\title{
Characterisation results for Steiner triple systems and their application to edge-colourings of cubic graphs
}

\author{
Daniel Král** Edita Máčajová ${ }^{*} \quad$ Attila Pór ${ }^{\ddagger}$ \\ Jean-Sébastien Sereni ${ }^{\S}$
}

\begin{abstract}
It is known that a Steiner triple system is projective if and only if it does not contain the four-triple configuration $C_{14}$. We find three configurations such that a Steiner triple system is affine if and only if it does not contain one of these configurations. Similarly, we characterise Hall triple systems using two forbidden configurations.

Our characterisations have several interesting corollaries in the area of edge-colourings of graphs. A cubic graph $G$ is $S$-edge-colourable for a Steiner triple system $S$ if its edges can be coloured with points of $S$ in such a way that the points assigned to three edges sharing a vertex
\end{abstract}

\footnotetext{
*Institute for Theoretical Computer Science (ITI), Faculty of Mathematics and Physics, Charles University, Malostranské náměstí 25, 11800 Prague 1, Czech Republic. Email: kral@kam.mff.cuni.cz. Institute for Theoretical computer science is supported as project $1 \mathrm{M} 0545$ by Czech Ministry of Education.

$\dagger$ Department of Computer Science, Faculty of Mathematics, Physics and Informatics, Comenius University, Mlynská dolina, 84248 Bratislava, Slovakia. E-mail: macajova@dcs.fmph.uniba.sk. This author is partially supported by APVT, project no. 51-027604.

${ }_{\ddagger}^{\ddagger}$ Department of Applied Mathematics (KAM) and Theoretical Computer Science (ITI), Faculty of Mathematics and Physics, Charles University, Malostranské náměstí 25, 11800 Prague 1, Czech Republic. E-mail: por@kam.mff.cuni.cz. This author is supported by the Hungarian National Foundation Grant T 046246.

$\S$ Department of Applied Mathematics (KAM) and Theoretical Computer Science (ITI), Faculty of Mathematics and Physics, Charles University, Malostranské náměstí 25, 11800 Prague 1, Czech Republic. E-mail: sereni@kam.mff.cuni.cz. This author is supported by EU project IST FET AEOLUs.
} 
form a triple in $S$. Among others, we show that all cubic graphs are $S$-edge-colourable for every non-projective non-affine point-transitive Steiner triple system $S$.

$M S C: 05 B 07,05 C 15$.

\section{Introduction}

Steiner triple systems form a classical notion in combinatorial design theory. Recall that a Steiner triple system $S$ is formed by $n$ points and several triples such that every two distinct points are contained in exactly one common triple. Steiner triple systems are simply-defined though complex and diverse combinatorial designs. One of the most classical results asserts the existence of a Steiner triple system with $n$ points whenever $n=1,3(\bmod 6), n \geq 3$. The amount of results on Steiner triple systems is enormous and a separate monograph [2] on the topic has recently appeared.

There are several prominent classes of Steiner triple systems. Among the most important ones are projective and affine Steiner triple systems. A projective Steiner triple system $\mathrm{PG}(d, 2)$ is the Steiner triple system with $2^{d+1}-1$ points corresponding to non-zero $(d+1)$-dimensional vectors over $\mathbb{Z}_{2}$ for $d \geq 1$. Three such vectors form a triple of $\operatorname{PG}(d, 2)$ if they sum to the zero vector. The smallest Steiner triple system is the projective system PG(1,2), comprised of three points forming a single triple. It is is referred to as the trivial Steiner triple system, while larger Steiner triple systems are called non-trivial. The smallest non-trivial projective Steiner triple system is $\mathrm{PG}(2,2)$, the Fano plane, which is denoted by $S_{7}$. An affine Steiner triple system $\mathrm{AG}(d, 3)$ is the Steiner triple system with $3^{d}$ points corresponding to $d$-dimensional vectors over $\mathbb{Z}_{3}$ for $d \geq 1$. Three such vectors form a triple of $\operatorname{AG}(d, 3)$ if they sum to the zero vector. The smallest affine Steiner triple system, $\mathrm{AG}(1,3)$, is isomorphic to the trivial Steiner triple system. The smallest non-trivial affine Steiner triple system, $\operatorname{AG}(2,3)$, is the unique Steiner triple system with nine points and is denoted by $S_{9}$.

It is natural to ask whether these two classes of Steiner triple systems can be characterised in terms of well-described forbidden substructures (as for instance, it is known that a graph is planar if and only if it does not contain a subdivision of one of the graphs $K_{3,3}$ or $K_{5}$ ). To be more precise, a configuration $C$ is formed by points and triples such that each pair of points is in at most one of the triples, and a Steiner triple system $S$ contains $C$ if 


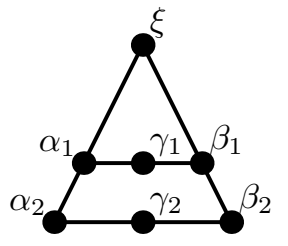

(a)

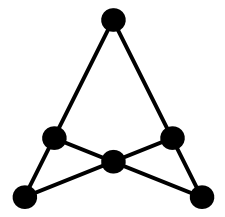

(b)

Figure 1: (a) The configuration $C_{14}$ and (b) the Pasch configuration $C_{16}$.

there is an injective mapping of the points of $C$ to the points of $S$ such that triples of points of $C$ are mapped to triples of points of $S$.

The solution to the just stated problem is easy to find for the class of projective Steiner triple systems. Two four-triple configurations play important roles in this characterisation: the first one is the configuration $C_{14}$ formed by seven distinct points $\xi, \alpha_{1}, \alpha_{2}, \beta_{1}, \beta_{2}, \gamma_{1}$ and $\gamma_{2}$ together with four triples $\left\{\xi, \alpha_{1}, \alpha_{2}\right\},\left\{\xi, \beta_{1}, \beta_{2}\right\},\left\{\alpha_{1}, \beta_{1}, \gamma_{1}\right\}$ and $\left\{\alpha_{2}, \beta_{2}, \gamma_{2}\right\}$; see Figure $1(a)$. The second one is the configuration $C_{16}$, known as the Pasch configuration, which is formed by six points and four triples; see Figure $1(b)$.

Clearly, the configuration $C_{14}$ cannot be contained in a projective Steiner triple system. The converse is also true: Stinson and Wei [12] established that a Steiner triple system $S$ with $n$ points is projective if and only if it contains $\frac{1}{24} n(n-1)(n-3)$ distinct copies of the Pasch configuration. By a counting argument given in [5], if $S$ contains less than $\frac{1}{24} n(n-1)(n-3)$ copies of the Pasch configuration, then it must contain a configuration isomorphic to $C_{14}$. We state this observation as a separate theorem.

Theorem 1 (Grannell et al. [5] and Stinson et al. [12]). A Steiner triple system $S$ is projective if and only if it contains no configuration $C_{14}$.

However, we were not able to find such a simple argument characterising affine Steiner triple systems in the literature. In Section 5, we show that a Steiner triple system is affine unless it contains one of the three configurations depicted in Figure 2, namely the Pasch configuration $C_{16}$, the configuration $C_{S}^{1}$ and the configuration $C_{S}^{2}$ (the last two configurations are obtained from the squashed square configuration $C_{S}$ introduced in Section 2).

Still a finer distinction between affine and non-affine Steiner triple systems can be achieved. A prominent class of Steiner triple systems are Hall triple 

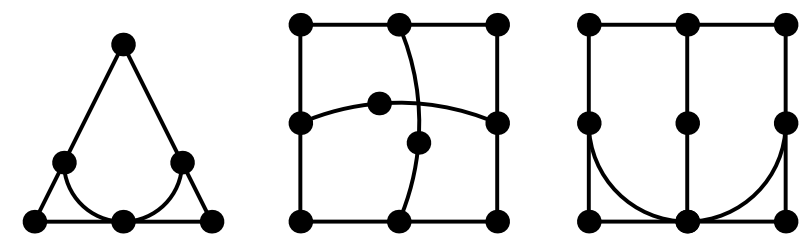

Figure 2: The three forbidden configurations for affine Steiner triple systems: the configurations $C_{16}, C_{S}^{1}$ and $C_{S}^{2}$ (from left to right).

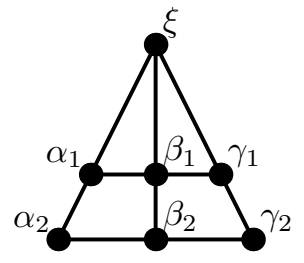

$(a)$

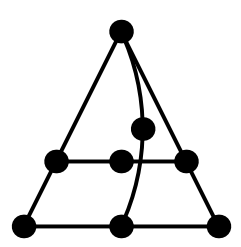

(b)

Figure 3: (a) The mitre configuration and $(b)$ the anti-mitre configuration $C_{A}$.

systems: a Steiner triple system $S$ is a Hall triple system if for every point $x$ of $S$, there exists an automorphism of $S$ that is involution and its only fixed point is $x$. Hall [7] showed that a Steiner triple system is a Hall triple system if and only if every Steiner triple system induced by the points of two nondisjoint triples of $S$ is isomorphic to $S_{9}$. Recall that the Steiner triple system induced by a set $X$ of the points of $S$ is the smallest Steiner triple system $S^{\prime}$ such that $S^{\prime}$ contains all the points of $X$ and all triples of $S^{\prime}$ are also triples of $S$. Hence, Hall triple systems look "locally" as affine Steiner triple systems and it can seem hard to distinguish these two classes in terms of forbidden substructures. Also note that there are examples of Hall triple systems that are not affine Steiner triple systems for every $n=3^{d}, d \geq 4$, unlike in the case of projective Steiner triple systems: if every two non-disjoint triples of $S$ induce a Steiner triple system isomorphic to $S_{7}$, then $S$ must be projective.

It is known that an $n$-point Steiner triple system is a Hall triple system if and only if it contains $\frac{n(n-1)(n-3)}{12}$ configurations isomorphic to the mitre configuration [2] which is depicted in Figure 3(a) (the points are labelled for future references). Since no Steiner triple system can contain more than 
$\frac{n(n-1)(n-3)}{12}$ copies of the mitre configuration, Hall triple systems are those which contains the largest number of configurations isomorphic to the mitre configuration.

Let us turn back to our results. As we mentioned, a Steiner triple system $S$ is affine if and only if it contains neither of the configurations $C_{16}, C_{S}^{1}$ and $C_{S}^{2}$. None of Hall triple systems can contain the configuration $C_{16}$ or the anti-mitre configuration $C_{A}$ (see Figure $3(b)$ ) as neither of them is contained in the Steiner triple system $S_{9}$. The converse is also true as we show in Section 5: a Steiner triple system is a Hall triple system if and only if it does not contain the configuration $C_{16}$ or $C_{A}$, and a Hall triple system is affine if and only if it contains none of the configuration $C_{S}^{1}$ and $C_{S}^{2}$. With the additional assumption that a Steiner triple system $S$ is not projective, we can remove the configuration $C_{16}$ from the list of forbidden configurations both for affine Steiner triple systems and Hall triple systems. We also note that every Steiner triple system containing the configuration $C_{A}$ also contains one of the configurations $C_{S}^{1}$ and $C_{S}^{2}$ (this seems to be a non-trivial fact which we state as Lemma 6).

\subsection{Edge-colourings of cubic graphs}

Edge-colourings of cubic (bridgeless) graphs form a prominent topic in graph theory because of its close relation to deep and important problems such as Four Colour Theorem, Cycle Double Cover Conjecture and many others. By Vizing's theorem [14], the edges of every cubic graph can be coloured with three or four colours in such a way that the edges meeting at the same vertex receive distinct colours. Non-trivially connected cubic graphs (usually the cyclic 4-edge-connectivity is required) such that their edges cannot be coloured with three colours are called snarks.

Archdeacon [1] proposed to study edge-colourings of cubic graphs by the points of Steiner triple systems. Steiner triple systems seem to be general enough to "edge-colour" most of cubic graphs and still well-structured enough to provide us with new results on cubic graphs. In particular, this notion extends the notion of the ordinary edge-colourings among the lines of wellstudied locally injective homomorphisms.

The points of a Steiner triple system $S$ are assigned to the edges of a cubic graph $G$ in such a way that the edges incident with the same vertex are assigned three distinct points that form a triple of $S$. Edge-colourings with this property are called $S$-edge-colourings and $G$ is said to be $S$-edge- 
colourable. A natural question is for which cubic graphs $G$ and which Steiner triple systems $S$, there exists an $S$-edge-colouring of $G$. In particular, whether there exists a Steiner triple system $S$ such that every simple cubic graph (bridgeless or not) is $S$-edge-colourable; such a system $S$ is called universal.

Grannell et al. [4] established the existence of a universal Steiner triple system with 381 points. Later, Pál and Škoviera [11] showed that there exists a universal Steiner triple system with 21 points (the system they considered is the direct product of the Fano plane and the trivial Steiner triple system). One of the corollaries of our results is the existence of a universal Steiner triple system with 13 points. Let us note that no Steiner triple system with less than 13 points can be universal [8].

We now survey further results on edge-colourings of cubic graphs with points of Steiner triple systems. Let us emphasise that all cubic graphs that we consider in this paper are connected and they can contain bridges unless stated otherwise. On the other hand, they never contain loops or parallel edges. This assumption does not decrease generality of our results: a cubic graph with a loop does not have an $S$-edge-colouring for any Steiner triple system $S$ since the points assigned to the edges incident with the vertex with the loop cannot form a triple in $S$. If a cubic graph $G$ contains a pair of parallel edges $e_{1}$ and $e_{2}$ between two vertices $v_{1}$ and $v_{2}$, let $G^{\prime}$ be the graph obtained from $G$ by removing $e_{1}$ and $e_{2}$ and identifying the other edges incident with $v_{1}$ and $v_{2}$. It is straightforward to verify that $G$ is $S$-edgecolourable if and only if $G^{\prime}$ is. Hence, we can subsequently eliminate the pairs of all parallel edges in a cubic graph. Note that loops can appear during this elimination process yielding that the original graph is not $S$-edge-colourable for any Steiner triple system $S$.

$\mathrm{Fu}[3]$ showed that every bridgeless cubic graph of order at most 189 and of genus at most 24 are $S_{7}$-edge-colourable. A stronger result was obtained by Holroyd and Škoviera [8] who showed that a cubic graph $G$ is $S$-edgecolourable for a non-trivial projective Steiner triple system $S$ if and only if it is bridgeless. In particular, all bridgeless cubic graphs are $S_{7}$-edge-colourable. The condition on $G$ being bridgeless can be easily seen to be necessary since an edge-colouring of $G$ with the points of a projective Steiner triple system $\operatorname{PG}(d, 2)$ can be viewed as a nowhere-zero flow over $\mathbb{Z}_{2}^{d+1}$. It is well-known that a graph has a nowhere-zero flow if and only if it is bridgeless.

A characterisation of cubic graphs that are $S$-edge-colourable for a nonprojective Steiner triple system $S$ has been offered as a conjecture (see Conjecture 1). One of the obstacles for the existence of an $S$-edge-colouring is 
the notion of a bipartite end which we now introduce. If a cubic graph $G$ has bridges it can be split along its bridges into 2-connected blocks, each incident with one or more bridges. Each bridge is split into two half-edges, each half-edge incident with one of the blocks. Note that some blocks can be formed by a single vertex incident with three half-edges; such blocks are called trivial. A block incident with a single half-edge is called end. Let $H$ be an end of a cubic graph and $H^{\prime}$ the graph obtained from $H$ by suppressing the vertex incident with the half-edge. Hence, $H^{\prime}$ is a bridgeless cubic graph which can contain a single pair of parallel edges (one of those is the contracted edge). We say that $H$ is a bipartite end if the graph $H^{\prime}$ is bipartite, $H$ is a 3-edge-colourable end if $H^{\prime}$ is 3-edge-colourable (in the usual sense), and $H$ is hamiltonian if $H^{\prime}$ has a Hamilton cycle avoiding the edge obtained by suppressing the vertex incident with the half-edge.

We can now state the conjectured characterisation of cubic graphs that are $S$-edge-colourable with a Steiner triple system $S$.

Conjecture 1 (Holroyd and Škoviera [8], Conjecture 1.4). Let $S$ be a nonprojective Steiner triple system. A cubic graph $G$ is $S$-edge-colourable unless $G$ has a bipartite end and $S$ is affine.

If a cubic graph $G$ has a bipartite end $H$ and a Steiner triple system $S$ is affine, an easy linear algebra argument yields that the two edges of $H$ incident with the bridge must be coloured with the same point of $S$ [8]. Hence, $G$ cannot be $S$-edge-coloured. The conjecture of Holroyd and Škoviera asserts this to be the only obstacle for the existence of an $S$-edge-colouring unless $S$ is projective. A counter-example to Conjecture 1 based on altering a projective Steiner triple system has been found very recently by Griggs and Máčajová [6]. Its structure suggests that Conjecture 1 is maybe not far from the truth, since the constructed counter-example is obtained through a simple modification of a projective Steiner triple system (an exceptional system in the conjecture).

As a corollary of our characterisation results on Steiner triple systems, we can show that Conjecture 1 is true when restricted to point-transitive Steiner triple systems. A Steiner triple system is point-transitive if for every two points $x$ and $y$ of $S$, there exists an automorphism of $S$ that maps $x$ to $y$. In fact, for non-trivial point-transitive Steiner triple systems $S$, we can characterise cubic graphs $G$ that are $S$-edge-colourable: if $S$ is projective, $G$ is $S$-edge-colourable if and only if $G$ is bridgeless (this follows from the results of [8]). If $S$ is affine, $G$ is $S$-edge-colourable if and only if $G$ has 
no bipartite end (this solves an open problem from $[8,11]$ whether every cubic graph with no bipartite end is $\mathrm{AG}(2,3)$-edge-colourable). Finally, if $S$ a non-trivial point-transitive Steiner triple system that is neither projective nor affine, then $G$ is always $S$-edge-colourable.

Since there exists a point-transitive Steiner triple system with 13 vertices that is neither projective nor affine, we can infer from our results the existence of a universal 13-point Steiner triple system. Since the only smaller Steiner triple systems are the trivial Steiner triple system, the projective Steiner triple system $S_{7}=\mathrm{PG}(2,2)$ and the affine Steiner triple system $S_{9}=\mathrm{AG}(2,3)$, the point-transitive Steiner triple system with 13 points is the universal Steiner triple system with the smallest number of points (this solves an open problem from [4] to determine the number of points of the smallest universal Steiner triple system).

\section{Notation}

In this section, we introduce some additional notation related to Steiner triple systems. If $S$ is a Steiner triple system, we find convenient to have a special notation for the point $z$ forming a triple with two given distinct points $x$ and $y$ : such point $z$ is denoted by $x \oplus y$ throughout the paper. For instance, in $C_{14}$ depicted in Figure $1(a), \xi=\beta_{1} \oplus \beta_{2}$. Note that the operation $\oplus$ is commutative and need not be associative, i.e., the points $x_{1} \oplus\left(x_{2} \oplus x_{3}\right)$ and $\left(x_{1} \oplus x_{2}\right) \oplus x_{3}$ could be distinct.

The set $X$ of points of a Steiner triple system $S$ is said to be independent if for every $x \in X$, the Steiner triple system $S^{\prime}$ induced by $X \backslash\{x\}$ in $S$ does not contain $x$. For instance, a set $X$ of points of an affine Steiner triple system is independent if and only if $X$ is affinely independent over $\mathbb{Z}_{3}$.

We now introduce another notion of containment of configurations in Steiner triple systems and relate it to the standard notion. Let $C$ be a configuration, as defined earlier, with a distinguished pair $(a, b)$ of its points. As an example, consider the squashed square configuration $C_{S}$ depicted in Figure 4 with $a=x_{\alpha \beta}$ and $b=x_{\gamma \delta}$. We say that a Steiner triple system $S$ homomorphically contains the configuration $C$ if there exists a mapping $\varphi$ of points of $C$ to the points of $S$ such that every triple of $C$ is injectively mapped onto a triple of $S$ and $\varphi(a) \neq \varphi(b)$. Note that we do not require the mapping $\varphi$ to be injective but we require that no two points of the same triple of $C$ are mapped to the same point of $S$ and that the points $a$ and $b$ 


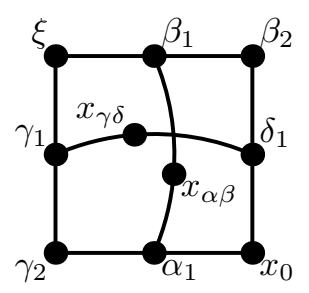

Figure 4: The squashed square configuration $C_{S}$. Note that it is required that $\varphi\left(x_{\alpha \beta}\right) \neq \varphi\left(x_{\gamma \delta}\right)$ but the other pairs of points can coincide in a Steiner triple system.

are mapped to distinct points of $S$.

In Sections 3 and 4, when proving our characterisation theorems, it will be more convenient to show that a given Steiner triple system homomorphically contains $C_{S}$, rather than it contains one of the configurations $C_{S}^{1}$ and $C_{S}^{2}$. Let us realise that the two statements are equivalent.

Lemma 2. A Steiner triple system $S$ homomorphically contains the squashed square configuration $C_{S}$ if and only if $S$ contains the configuration $C_{S}^{1}$ or the configuration $C_{S}^{2}$.

Proof. If $S$ contains the configuration $C_{S}^{1}$ or the configuration $C_{S}^{2}$, then $S$ homomorphically contains $C_{S}$. In the rest, we focus on proving the converse implication. Let $X$ be the set of points of the squashed square configuration $C_{S}$; the points of $C_{S}$ are denoted as in Figure 4. Assume that $S$ homomorphically contains the squashed square configuration $C_{S}$ and let $\varphi$ be the mapping from $X$ to the points of $C_{S}$ as in the definition of homomorphical containment.

We first show that $\varphi(\xi)$ is distinct from $\varphi(x)$ for every $x \in X \backslash\{\xi\}$. By symmetry, it is enough to consider that $\varphi(\xi)$ would be equal to $\varphi\left(\alpha_{1}\right), \varphi\left(x_{0}\right)$ or $\varphi\left(x_{\alpha \beta}\right)$ and obtain a contradiction. If $\varphi(\xi)=\varphi\left(\alpha_{1}\right)$, then $\varphi\left(x_{0}\right)=\varphi\left(\gamma_{1}\right)$ since $\gamma_{2}=\xi \oplus \gamma_{1}=\alpha_{1} \oplus x_{0}$ (in more detail, since $\varphi(\xi)=\varphi\left(\alpha_{1}\right)$ and $S$ contains the triples $\left\{\varphi\left(\gamma_{2}\right), \varphi(\xi), \varphi\left(\alpha_{1}\right)\right\}$ and $\left\{\varphi\left(\gamma_{2}\right), \varphi\left(\alpha_{1}\right), \varphi\left(x_{0}\right)\right\}$, it must also hold that $\left.\varphi\left(\alpha_{1}\right)=\varphi\left(x_{0}\right)\right)$. Similarly, the equality $\beta_{1}=\xi \oplus \beta_{2}=\alpha_{1} \oplus x_{\alpha \beta}$ implies that $\varphi\left(\beta_{2}\right)=\varphi\left(x_{\alpha \beta}\right)$, and $\delta_{1}=x_{0} \oplus \beta_{2}=\gamma_{1} \oplus x_{\gamma \delta}$ yields that $\varphi\left(\beta_{2}\right)=\varphi\left(x_{\gamma \delta}\right)$. We infer that $\varphi\left(x_{\alpha \beta}\right)=\varphi\left(x_{\gamma \delta}\right)=\varphi\left(\beta_{2}\right)$ which is impossible.

If it held $\varphi(\xi)=\varphi\left(x_{0}\right)$, then it would also hold that $\varphi\left(\alpha_{1}\right)=\varphi\left(\gamma_{1}\right)$ and $\varphi\left(\beta_{1}\right)=\varphi\left(\delta_{1}\right)$. Consequently, $\varphi\left(x_{\alpha \beta}\right)=\varphi\left(x_{\gamma \delta}\right)$ contrary to the definition of 
homomorphical containment.

Finally, if $\varphi(\xi)=\varphi\left(x_{\alpha \beta}\right)$, we obtain $\varphi\left(\alpha_{1}\right)=\varphi\left(\beta_{2}\right)$. From this it follows that $\varphi\left(x_{\alpha \beta}\right)=\varphi\left(x_{\gamma \delta}\right)$ similarly to the case where $\varphi(\xi)=\varphi\left(\alpha_{1}\right)$ with $\beta_{2}$ playing the role of $\xi$.

If $\varphi$ maps $X$ to ten distinct points of $S$, i.e., $\varphi$ is injective, then $S$ contains the configuration $C_{S}^{1}$. Otherwise, two of the points of $X$ are mapped to the same point of $S$. As the points $\xi, x_{0}, \beta_{2}$ and $\gamma_{2}$ are mapped to distinct points of $S, \varphi\left(\alpha_{1}\right) \neq \varphi\left(\delta_{1}\right)$ (otherwise, $x_{0}=\alpha_{1} \oplus \gamma_{2}=\delta_{1} \oplus \beta_{2}$ implies that $\left.\varphi\left(\beta_{2}\right)=\varphi\left(\gamma_{2}\right)\right)$. By the symmetry, we can assume that $\varphi\left(\alpha_{1}\right)=\varphi\left(x_{\gamma \delta}\right)$. It is now straightforward to check that no other two points of $X$ can be mapped by $\varphi$ to the same point of $S$ and thus $S$ contains the configuration $C_{S}^{2}$. We conclude that if $S$ homomorphically contains the squashed-square configuration $C_{S}$, then $S$ contains one of the configurations $C_{S}^{1}$ and $C_{S}^{2}$.

\section{Forbidden configurations in Hall triple sys- tems}

We split the proof of our characterisation results into two parts. In this section, we deal with Hall triple systems and in the next section, we focus on non-Hall triple systems.

Lemma 3. Every Hall triple system $S$ that is not affine homomorphically contains the squashed-square configuration $C_{S}$.

Proof. Let us first introduce some additional notation that we use throughout the proof. Fix an arbitrary point of $S$; we refer to this point as to the zero and it is denoted by $\underline{0}$. The other points of $S$ are said to be non-zero.

For a set $M$ of non-zero points of $S, L(M)$ is the Steiner triple system induced by $M \cup\{\underline{0}\}$. If $M$ contains a single point, then $L(M)$ is isomorphic to the trivial Steiner triple system. Observe that if a set $M$ of non-zero points of $S$ is such that $a \notin L(M \backslash\{a\})$ for every $a \in M$, then it is independent, as defined in Section 2. Since $S$ is Hall, $L(M)$ is isomorphic to $S_{9}$ for every two-point set $M$ such that $M \cup\{\underline{0}\}$ is independent. On the other hand, since $S$ is not affine there exists an independent set $M$ such that $L(M)$ is not an affine Steiner triple system. Let $M=\left\{e_{1}, \ldots, e_{d}\right\}$ be an inclusion-wise minimal independent set for which $L(M)$ is not affine. Note that $d \geq 3$.

Let $V=\mathbb{Z}_{3}^{d}$ and let $V^{0}$ be the set of the vectors of $V$ with at least one coordinate equal to zero. Define a mapping $\phi: V^{0} \rightarrow L(M)$ as follows: the 


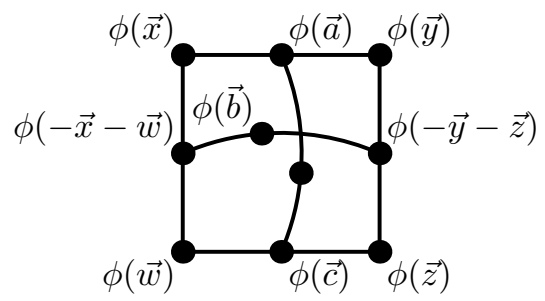

$(a)$

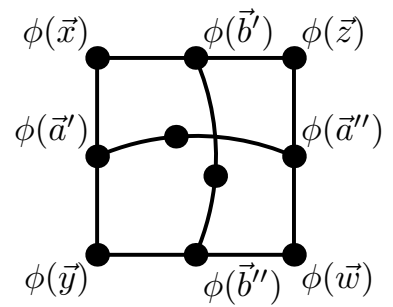

(b)

Figure 5: Two squashed-square configurations constructed in the proof of Lemma 3.

zero-vector is mapped to $\underline{0}$ and the unit vectors are mapped to the points of $M$. The mapping $\phi$ is then extended to the remaining vectors of $V^{0}$ in such a way that whenever the vectors $\vec{a}, \vec{b}$ and $\vec{c}$ have at least one common coordinate equal to zero and sum to zero, then $\phi(\vec{a}), \phi(\vec{b})$ and $\phi(\vec{c})$ form a triple in $L(M)$. Since each of the systems $L\left(M \backslash\left\{e_{i}\right\}\right), i=1, \ldots, d$, is affine, such an extension of $\phi$ to $V^{0}$ is well-defined and unique.

Since $L(M)$ is not affine, the mapping cannot be extended to the whole set $V$ in such a way that each triple of vectors summing to zero is mapped to a triple of $L(M)$. There could be several obstacles for the existence of such an extension. The simplest one is that there exist three vectors $\vec{a}, \vec{b}$ and $\vec{c}$ of $V^{0}$ such that $\vec{a}+\vec{b}+\vec{c}=\overrightarrow{0}$ but the points $\phi(\vec{a}), \phi(\vec{b})$ and $\phi(\vec{c})$ do not form a triple in $L(M)$. By the definition of $\phi$, the vectors $\vec{a}, \vec{b}$ and $\vec{c}$ cannot have a common coordinate equal to zero. Hence, there exist three distinct indices $i$, $j$ and $k$ such that $\vec{a}_{i}=\vec{b}_{j}=\vec{c}_{k}=0$ but the coordinates $\vec{a}_{j}, \vec{a}_{k}, \vec{b}_{i}, \vec{b}_{k}, \vec{c}_{i}$ and $\vec{c}_{j}$ are non-zero.

We now define four vectors $\vec{w}, \vec{x}, \vec{y}$ and $\vec{z}$ based on the vectors $\vec{a}, \vec{b}, \vec{c}$. The $i$-th coordinates of the vectors are $\vec{x}_{i}=\vec{y}_{i}=\vec{z}_{i}=0$ and $\vec{w}_{i}=-\vec{c}_{i}$; the $j$-th coordinates are $\vec{x}_{j}=\vec{w}_{j}=0, \vec{y}_{j}=-\vec{a}_{j}$ and $\vec{z}_{j}=-\vec{c}_{j}$; and the $k$-th coordinates are $\vec{x}_{k}=\vec{y}_{k}=\vec{a}_{k}$ and $\vec{w}_{k}=\vec{z}_{k}=0$. The remaining coordinates are set as $\vec{x}_{t}=-\vec{a}_{t}, \vec{y}_{t}=0$, and $\vec{w}_{t}=\vec{z}_{t}=\vec{c}_{t}$ for $t \notin\{i, j, k\}$. Note that $\vec{a}+\vec{x}+\vec{y}=\overrightarrow{0}$ and $\vec{c}+\vec{w}+\vec{z}=\overrightarrow{0}$. In addition, the vectors $\vec{x}$ and $\vec{w}$ have a common coordinate equal to zero, and similarly the pairs of vectors $\vec{x}$ and $\vec{y}$, $\vec{y}$ and $\vec{z}$, and $\vec{w}$ and $\vec{z}$. Observe also that the $j$-th coordinate of $\vec{b}$ and $-(\vec{x}+\vec{w})$ is zero, and $\vec{b}+(-\vec{x}-\vec{w})+(-\vec{y}-\vec{z})=\overrightarrow{0}$. Since $\phi(\vec{b}) \neq \phi(\vec{a}) \oplus \phi(\vec{c})$ by our assumption, the configuration looks as depicted in Figure 5(a). Hence, we 
have exhibited the squashed-square configuration in $S$.

Assume now that for every triple $\vec{a}, \vec{b}$ and $\vec{c}$ of vectors of $V^{0}$ with $\vec{a}+\vec{b}+\vec{c}=$ $\overrightarrow{0}$, the points $\phi(\vec{a}), \phi(\vec{b})$ and $\phi(\vec{c})$ form a triple in $L(M)$. Our next aim is to try to extend $\phi$ to $V$ by defining $\phi(\vec{a})=\phi\left(\vec{a}^{\prime}\right) \oplus \phi\left(\vec{a}^{\prime \prime}\right)$ for vectors $\vec{a} \in V \backslash V^{0}$ and $\vec{a}^{\prime}, \vec{a}^{\prime \prime} \in V^{0}$ such that $\vec{a}+\vec{a}^{\prime}+\vec{a}^{\prime \prime}=\overrightarrow{0}$. This extension would be possible unless there existed four vectors $\vec{a}^{\prime}, \vec{a}^{\prime \prime}, \vec{b}^{\prime}, \vec{b}^{\prime \prime} \in V^{0}$ such that $\vec{a}^{\prime}+\vec{a}^{\prime \prime}=\vec{b}^{\prime}+\vec{b}^{\prime \prime}$ and $\phi\left(\vec{a}^{\prime}\right) \oplus \phi\left(\vec{a}^{\prime \prime}\right) \neq \phi\left(\overrightarrow{b^{\prime}}\right) \oplus \phi\left(\overrightarrow{b^{\prime \prime}}\right)$. Assume the existence of such vectors $\vec{a}^{\prime}, \vec{a}^{\prime \prime}, \overrightarrow{b^{\prime}}$ and $\vec{b}^{\prime \prime}$.

We next find four vectors $\vec{w}, \vec{x}, \vec{y}$ and $\vec{z}$ in $V^{0}$ such that $\vec{a}^{\prime}+\vec{x}+\vec{y}=\overrightarrow{0}$, $\overrightarrow{b^{\prime}}+\vec{x}+\vec{z}=\overrightarrow{0}, \vec{a}^{\prime \prime}+\vec{w}+\vec{z}=\overrightarrow{0}$ and $\overrightarrow{b^{\prime \prime}}+\vec{y}+\vec{w}=\overrightarrow{0}$; see Figure $5(b)$. Thus $S$ contains the squashed-square configuration. Note that $\vec{a}^{\prime}+\vec{a}^{\prime \prime}=\vec{b}^{\prime}+b^{\prime \prime} \notin V^{0}$ by our assumptions. In particular, the vectors $\vec{a}^{\prime}$ and $\vec{a}^{\prime \prime}$ do not have the same coordinate equal to zero and the same also holds for $\vec{b}^{\prime}$ and $\overrightarrow{b^{\prime \prime}}$. If we fix arbitrarily one of the coordinates of $\vec{w}$, say $\vec{w}_{i}$, then the $i$-th coordinates of the vectors $\vec{x}, \vec{y}$ and $\vec{z}$ are uniquely determined: $\vec{y}_{i}=-\left(\vec{b}_{i}^{\prime \prime}+\vec{w}_{i}\right), \vec{z}_{i}=-\left(\vec{a}_{i}^{\prime \prime}+\vec{w}_{i}\right)$ and $\vec{x}_{i}=-\left(\vec{a}_{i}^{\prime}+\vec{y}_{i}\right)=-\left(\vec{b}_{i}^{\prime}+\vec{z}_{i}\right)$ (the last two expressions are the same since $\left.\vec{a}_{i}^{\prime}+\vec{a}_{i}^{\prime \prime}=\vec{b}_{i}^{\prime}+\vec{b}_{i}^{\prime \prime}\right)$. Similarly, fixing the $i$-th coordinate of $\vec{x}$ determines the $i$-th coordinate of the vectors $\vec{y}, \vec{z}$ and $\vec{w}$.

First we assume that the vectors $\vec{a}^{\prime}$ and $\vec{b}^{\prime}$ have the same coordinate equal to zero, say $\vec{a}_{i}^{\prime}=\vec{b}_{i}^{\prime}=0$. We set $\vec{x}_{i}=0$. This determines the $i$-th coordinates of the vectors $\vec{w}, \vec{y}$ and $\vec{z}$. In particular, $\vec{y}_{i}=0$ and $\vec{z}_{i}=0$. Since $\vec{a}^{\prime \prime}, \vec{b}^{\prime \prime} \in V^{0}$, there exist $i^{\prime}$ and $i^{\prime \prime}$ such that $\vec{a}_{i^{\prime}}^{\prime \prime}=0$ and $\vec{b}_{i^{\prime \prime}}^{\prime \prime}=0$ (the indices $i^{\prime}$ and $i^{\prime \prime}$ need not be distinct). Since the vectors $\vec{a}^{\prime}$ and $\vec{a}^{\prime \prime}$ do not have a common coordinate equal to zero, $i \neq i^{\prime}$. Analogously, $i \neq i^{\prime \prime}$. Set $\vec{w}_{i^{\prime}}=0$ and $\vec{w}_{i^{\prime \prime}}=0$. The remaining coordinates of the vector $\vec{w}$ are chosen arbitrarily. This completely determines all the vectors $\vec{w}, \vec{x}, \vec{y}$ and $\vec{z}$. All the vectors $\vec{w}$, $\vec{x}, \vec{y}$ and $\vec{z}$ are in $V^{0}$ and they satisfy the above constraints. Analogously, we can handle the cases where the vectors $\vec{a}^{\prime}$ and $\vec{b}^{\prime \prime}, \vec{a}^{\prime \prime}$ and $\overrightarrow{b^{\prime}}$, or $\vec{a}^{\prime \prime}$ and $\vec{b}^{\prime \prime}$ have the same coordinate equal to zero.

We now assume that no pair of the vectors $\vec{a}^{\prime}, \vec{a}^{\prime \prime}, \vec{b}^{\prime}$ and $\vec{b}^{\prime \prime}$ have the same coordinate equal to zero. Let $i^{\prime}, i^{\prime \prime}, j^{\prime}$ and $j^{\prime \prime}$ be the indices such that $\vec{a}_{i^{\prime}}^{\prime}=0$, $\vec{a}_{i^{\prime \prime}}^{\prime \prime}=0, \vec{b}_{j^{\prime}}^{\prime}=0$ and $\vec{b}_{j^{\prime \prime}}^{\prime \prime}=0$. Choose $\vec{x}_{i^{\prime}}=\vec{x}_{j^{\prime}}=0$ and $\vec{w}_{i^{\prime \prime}}=\vec{w}_{j^{\prime \prime}}=0$. This determines the $i^{\prime}$-th, $i^{\prime \prime}$-th, $j^{\prime}$-th and $j^{\prime \prime}$-th coordinate of all the vectors $\vec{w}$, $\vec{x}, \vec{y}$ and $\vec{z}$. The remaining coordinates of the vector $\vec{x}$ are chosen arbitrarily and the remaining coordinates of the other vectors are determined by this choice. Again, we have obtained the configuration depicted in Figure $5(b)$.

Hence, we have excluded the existence of the vectors $\vec{a}^{\prime}, \vec{a}^{\prime \prime}, \overrightarrow{b^{\prime}}$ and $\overrightarrow{b^{\prime \prime}}$. 
Therefore, we conclude that the mapping $\phi$ can be extended to $V \backslash V^{0}$ in such a way that $\phi(\vec{x})=\phi\left(\vec{x}^{\prime}\right) \oplus \phi\left(\vec{x}^{\prime \prime}\right)$ for all vectors $\vec{x} \in V$ and $\vec{x}^{\prime}, \vec{x}^{\prime \prime} \in V^{0}$ with $\vec{x}+\vec{x}^{\prime}+\vec{x}^{\prime \prime}=\overrightarrow{0}$. Since $L(M)$ is not affine, there must exist three vectors $\vec{a}, \vec{b}$ and $\vec{c}$ in $V$ such that $\vec{a}+\vec{b}+\vec{c}=\overrightarrow{0}$ and $\phi(\vec{c}) \neq \phi(\vec{a}) \oplus \phi(\vec{b})$ for this (uniquely defined) mapping $\phi$.

If $\vec{a}=\overrightarrow{0}$, choose arbitrary two vectors $\vec{b}^{\prime}$ and $\vec{b}^{\prime \prime}$ in $V^{0}$ such that $\vec{b}+\overrightarrow{b^{\prime}}+\overrightarrow{b^{\prime \prime}}=$ $\overrightarrow{0}$. Hence, $\vec{c}+\left(\vec{a}+\overrightarrow{b^{\prime}}\right)+\left(\vec{a}+\overrightarrow{b^{\prime \prime}}\right)=\overrightarrow{0}$ and both $\vec{a}+\overrightarrow{b^{\prime}}$ and $\vec{a}+\overrightarrow{b^{\prime \prime}}$ are contained in $V^{0}$. Since $S$ is Hall and $\phi(\vec{x})=\phi\left(\vec{x}^{\prime}\right) \oplus \phi\left(\vec{x}^{\prime \prime}\right)$ for all vectors $\vec{x} \in V$ and $\vec{x}^{\prime}, \vec{x}^{\prime \prime} \in V^{0}$ with $\vec{x}+\vec{x}^{\prime}+\vec{x}^{\prime \prime}=\overrightarrow{0}$, it follows that $\phi(\vec{c})=\phi(\vec{a}) \oplus \phi(\vec{b})$, a contradiction. Hence, $\vec{a} \neq \overrightarrow{0}$.

Since the vectors $\vec{a}, \vec{b}$ and $\vec{c}$ are distinct, there exists $i$ such that $\vec{a}_{i}, \vec{b}_{i}$ and $\vec{c}_{i}$ are distinct. By symmetry, we can assume that $\vec{a}_{i}=0$. We aim to define four vectors $\vec{w}, \vec{x}, \vec{y}$ and $\vec{z}$ that would form the configuration depicted in Figure $5(a)$. Set $\vec{x}_{i}=\vec{y}_{i}=\vec{z}_{i}=0$ and $\vec{w}_{i}=-\vec{c}_{i}$. Let $j$ be the index such that $\vec{a}_{j} \neq 0$ and set $\vec{x}_{j}=\vec{w}_{j}=0, \vec{y}_{j}=-\vec{a}_{j}$ and $\vec{z}_{j}=-\vec{c}_{j}$. The remaining coordinates of the vectors $\vec{w}, \vec{x}, \vec{y}$ and $\vec{z}$ are chosen in such a way that $\vec{a}+\vec{x}+\vec{y}=\overrightarrow{0}$ and $\vec{c}+\vec{w}+\vec{z}=\overrightarrow{0}$. Observe that $\vec{x}+\vec{w} \in V^{0}$ and $\vec{y}+\vec{z} \in V^{0}$. Hence, $\phi(\vec{b})=\phi(-\vec{x}-\vec{w}) \oplus \phi(-\vec{y}-\vec{z})$ since $\vec{b}+(-\vec{x}-\vec{w})+(-\vec{y}-\vec{z})=\overrightarrow{0}$. We conclude that the constructed configuration matches that depicted in Figure 5(a). This finishes the proof that if the mapping $\phi$ cannot be extended to the whole set $V$, then $S$ homomorphically contains the squashed-square configuration. Hence, the proof of the lemma is complete.

\section{Forbidden configurations in other Steiner triple systems}

In the previous section, we have dealt with Hall triple systems, and in this section we consider non-Hall triple systems. In the next section, the results

of the previous and this section are combined to obtain characterisations of affine Steiner triple systems and Hall triple systems in terms of forbidden subconfigurations.

Our aim is to show that every non-projective Steiner triple system that is not Hall contains the anti-mitre configuration $C_{A}$, depicted in Figure $3(b)$. As the first step, we establish that if a Steiner triple system $S$ does not contain $C_{A}$, then every three independent points of $S$ induce a system isomorphic to $S_{7}$ or $S_{9}$. 


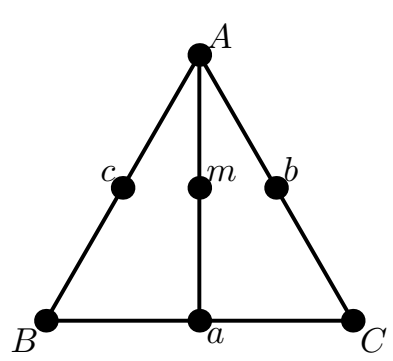

(a)

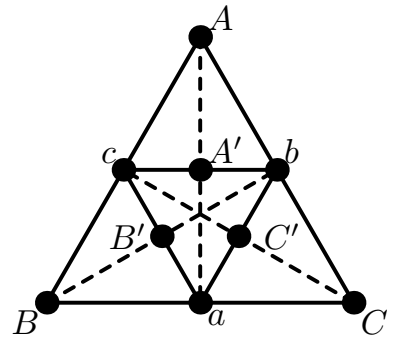

(b)

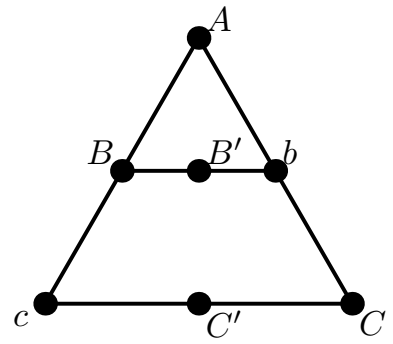

$(c)$

Figure 6: The configurations considered in the proof of Lemma 4.

Lemma 4. If a Steiner triple system $S$ does not contain the anti-mitre configuration, then every three independent points of $S$ induce a Steiner triple system isomorphic to $S_{7}$ or $S_{9}$.

Proof. Let $A, B$ and $C$ be three independent points of $S$ and let $a=B \oplus C$, $b=A \oplus C$ and $c=A \oplus B$; see Figure $6(a)$. Note that all the points $A, B$, $C, a, b$ and $c$ are mutually distinct. We next consider two cases.

- The points $a, b$ and $c$ form a triple of $S$.

Let $m=A \oplus a$. If $m \oplus c$ is neither $b$ nor $C$, we obtain a configuration isomorphic to the anti-mitre configuration. Since the points $a, b$ and $c$ form a triple in $S, m \oplus c$ cannot be $b$. Hence, $m \oplus c=C$. A symmetric argument yields that $m \oplus b=B$. So the points $A, B$ and $C$ induce a Steiner triple system isomorphic to $S_{7}$.

- The points $a, b$ and $c$ are independent.

Let $A^{\prime}=b \oplus c, B^{\prime}=a \oplus c$ and $C^{\prime}=a \oplus b$. Since $a, b$ and $c$ are independent, all the points $A, B, C, a, b, c, A^{\prime}, B^{\prime}$ and $C^{\prime}$ are mutually distinct. Since $S$ contains no anti-mitre configuration, the point $A^{\prime}$ is either $a$ or $A \oplus a$. We have just excluded the former case. Hence, $A^{\prime}=A \oplus a$. By the symmetry, we deduce that $B^{\prime}=B \oplus b$ and $C^{\prime}=C \oplus c$.

Therefore we obtain the configurations depicted in Figures 6(b) and (c). Again, since $S$ does not contain the anti-mitre configuration, the point $B^{\prime}$ is equal to either $C^{\prime}$ or $A \oplus C^{\prime}$. Since the points $B^{\prime}$ and $C^{\prime}$ are distinct, it holds that $B^{\prime}=A \oplus C^{\prime}$. By the symmetry, $B=A^{\prime} \oplus C^{\prime}$ and 


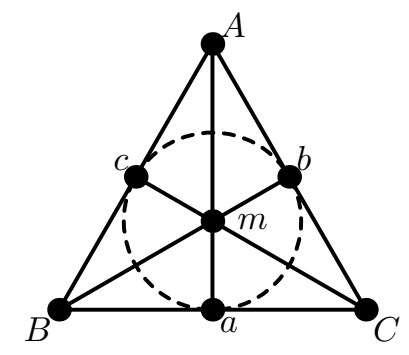

Figure 7: Notation used in the proof of Lemma 5.

$C=A^{\prime} \oplus B^{\prime}$. We conclude that the points $A, B, C, a, b, c, A^{\prime}, B^{\prime}$ and $C^{\prime}$ induce a Steiner triple system isomorphic to $S_{9}$.

Using Lemma 4, we can now show that every non-projective Steiner triple that is not Hall contains the anti-mitre configuration.

Lemma 5. Every Steiner triple system $S$ that is not projective and that is not a Hall triple system contains the anti-mitre configuration $C_{A}$.

Proof. Assume for the sake of contradiction that $S$ is neither a projective Steiner triple system nor a Hall triple system and yet does not contain the configuration $C_{A}$. By Lemma 4 , every three independent points induce a Steiner triple system isomorphic to $S_{7}$ or $S_{9}$. If all such induced systems are isomorphic to $S_{7}$, then $S$ is projective. On the other hand, since $S$ is not a Hall triple system, all of them cannot be isomorphic to $S_{9}$ either. Hence, there exist four independent points $A, B, C$ and $D$ such that the points $A$, $B$ and $C$ induce a Steiner triple system $S^{\prime}$ isomorphic to $S_{7}$, and there exist two independent points $t$ and $t^{\prime}$ of $S^{\prime}$ such that the points $D, t$ and $t^{\prime}$ induce a Steiner triple system isomorphic to $S_{9}$.

The Steiner triple system induced by $A, B$ and $C$ is isomorphic to $S_{7}$. We set $a=B \oplus C, b=A \oplus C, c=A \oplus B$ and $m=A \oplus a=B \oplus b=C \oplus c$; see Figure 7. Let $D_{A}=A \oplus D, D_{B}=B \oplus D, D_{C}=C \oplus D, D_{a}=a \oplus D$, $D_{b}=b \oplus D, D_{c}=c \oplus D$ and $D_{m}=m \oplus D$. Note that all the seven points $D_{A}, \ldots, D_{m}$ are distinct and neither of them is contained in $S^{\prime}$.

We now colour the triples of $S^{\prime}$ with red and blue based on the following rule: if $\left\{t, t^{\prime}, t^{\prime \prime}\right\}$ is a triple of $S^{\prime}$ and the points $t, t^{\prime}$ and $D$ induce a Steiner triple system isomorphic to $S_{7}$, colour the triple $\left\{t, t^{\prime}, t^{\prime \prime}\right\}$ red; otherwise, the 


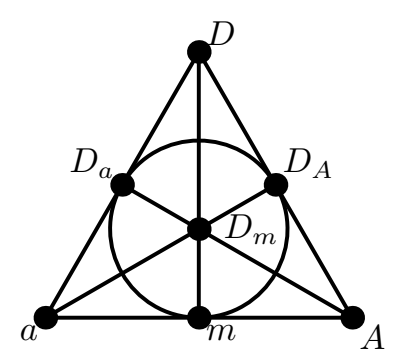

(a)

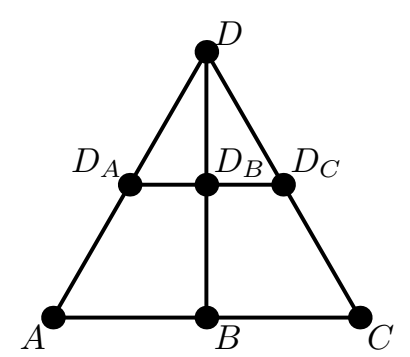

(d)

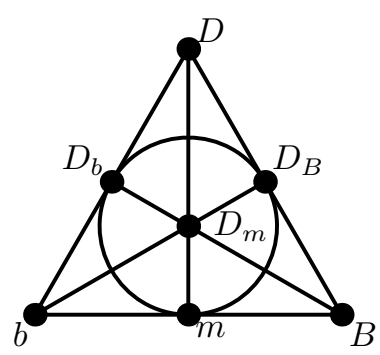

(b)

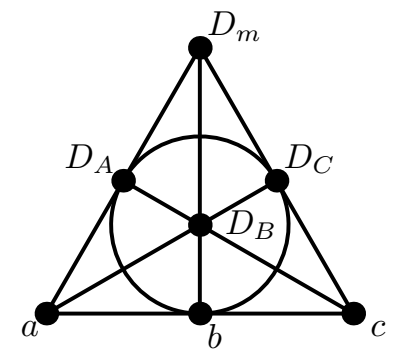

(e)

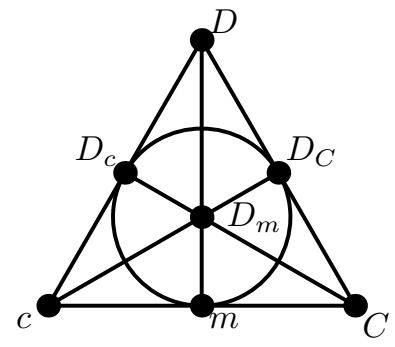

$(c)$

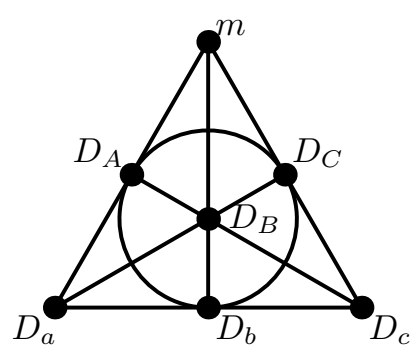

$(f)$

Figure 8: Configurations contained in $S$ in the case where all the sets $\{A, a, D\},\{B, b, D\}$ and $\{C, c, D\}$ induce Steiner triple systems isomorphic to $S_{7}$.

points induce a Steiner triple system isomorphic to $S_{9}$ and the triple $\left\{t, t^{\prime}, t^{\prime \prime}\right\}$ is coloured blue. This colouring is well-defined and by our assumptions, at least one of the seven triples of $S^{\prime}$ is blue. Observe now that $S^{\prime}$ contains a point such that all the three triples containing this point have the same colour. By symmetry, we can assume that $m$ is such a point and consider only the following two cases.

- The sets $\{A, a, D\},\{B, b, D\}$ and $\{C, c, D\}$ induce Steiner triple systems isomorphic to $S_{7}$.

Observe that $D_{A}=m \oplus D_{a}=D_{m} \oplus a, D_{B}=m \oplus D_{b}=D_{m} \oplus b$ and $D_{C}=m \oplus D_{c}=D_{m} \oplus c$; see Figure $8(a),(b)$ and $(c)$ respectively. Since there exist two points of $S^{\prime}$ that with $D$ induce a Steiner triple system isomorphic to $S_{9}$, we can assume by symmetry that the points $\{a, b, D\}$ induce a system isomorphic to $S_{9}$.

Note that the points $D_{A}, D_{B}$ and $D_{C}$ cannot form a triple: otherwise, the point $C$ would be contained in the Steiner triple system induced 
by $A, B$ and $D$ contradicting our choice of $A, B, C$ and $D$ as four independent points; see Figure $8(d)$. Therefore, the Steiner triple system induced by $D_{m}, a$ and $b$ is not affine and thus it is isomorphic to $S_{7}$; see Figure 8(e). Similarly, the Steiner triple system induced by $m, D_{a}$ and $D_{b}$ is isomorphic to $S_{7}$ (Figure $8(f)$ ). This readily implies that the points $b$ and $D_{b}$ must be the same since they both are the point $D_{A} \oplus D_{C}$. However, the points $b$ and $D_{b}$ must be distinct since they are contained in a common triple in $S$.

- The sets $\{A, a, D\},\{B, b, D\}$ and $\{C, c, D\}$ induce Steiner triple systems isomorphic to $S_{9}$.

Assume first that $D$ and every two points of $S^{\prime}$ induce a Steiner triple system isomorphic to $S_{9}$. In particular, the points $A, B$ and $D$ induce such a Steiner triple system and thus $(A \oplus D) \oplus(B \oplus D)=D_{A} \oplus D_{B}$ is equal to $c \oplus D=D_{c}$; see Figure $9(a)$. Let $x=m \oplus D_{A}=D_{m} \oplus a$, $y=m \oplus D_{B}=D_{m} \oplus b, z=m \oplus D_{C}=D_{m} \oplus c$ and $z^{\prime}=m \oplus D_{c}=D_{m} \oplus C$ (the points $x, y, z$ and $z^{\prime}$ exist since the configurations are isomorphic to $\left.S_{9}\right)$. See Figure $9(b),(c)$ and $(d)$.

Consider the Steiner triple system induced by the points $D_{m}, a$ and $b$. If this Steiner triple system is isomorphic to $S_{7}$, then $x \oplus y$ is the point $c$; otherwise, it is isomorphic to $S_{9}$ and $x \oplus y=z$. Consider now the Steiner triple system induced by the points $m, D_{A}$ and $D_{B}$. If it is isomorphic to $S_{7}$, then $x \oplus y=D_{A} \oplus D_{B}=D_{c}$; otherwise, it is isomorphic to $S_{9}$ and $x \oplus y$ is the point $z^{\prime}=m \oplus D_{c}=D_{m} \oplus C$. We conclude that $x \oplus y$ is the same point as two of the points $c, z, D_{c}$ and $z^{\prime}$. However, this is impossible since these points are mutually distinct as they form a Steiner triple system isomorphic to $S_{9}$. We conclude that $S^{\prime}$ contains two points that induce with $D$ a Steiner triple system isomorphic to $S_{7}$.

By symmetry, we can now assume that the points $a, b$ and $D$ induce a Steiner triple system isomorphic to $S_{7}$, see Figure 10. In particular, $b=D_{a} \oplus D_{c}$. Consider the Steiner triple system induced by the points $D_{m}, D_{a}$ and $D_{c}$. Since $D_{m} \oplus D_{a}=D_{A}$ and $D_{m} \oplus D_{c}=D_{C}$, the point $D_{A} \oplus D_{C}$ is either the point $b$ (if the considered Steiner triple system is isomorphic to $S_{7}$ ) or the point $D_{m} \oplus b$ (if it is isomorphic to $S_{9}$ ). By considering the Steiner triple system induced by the points $D, A$ and $C$, we derive that the point $D_{A} \oplus D_{C}$ is either $b$ or $D \oplus b$. The 


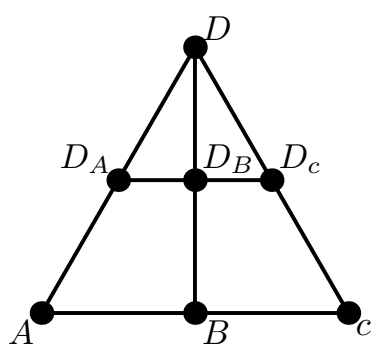

(a)

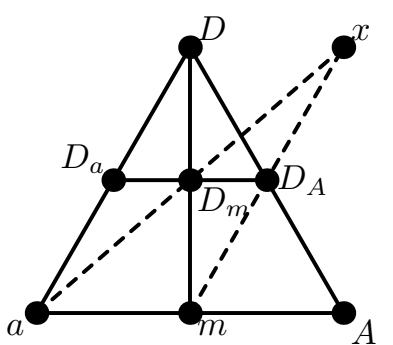

(b)

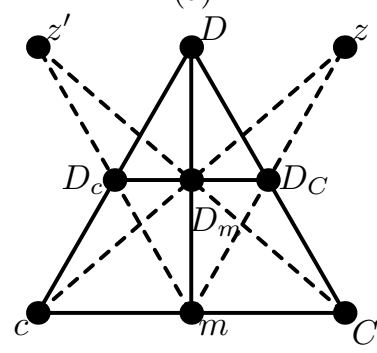

$(d)$

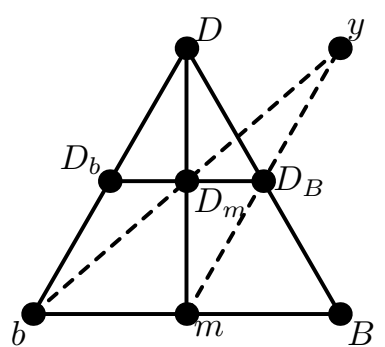

(c)

Figure 9: Configurations contained in $S$ in the case where $D$ and every two points of $S^{\prime}$ induce a Steiner triple systems isomorphic to $S_{9}$. 

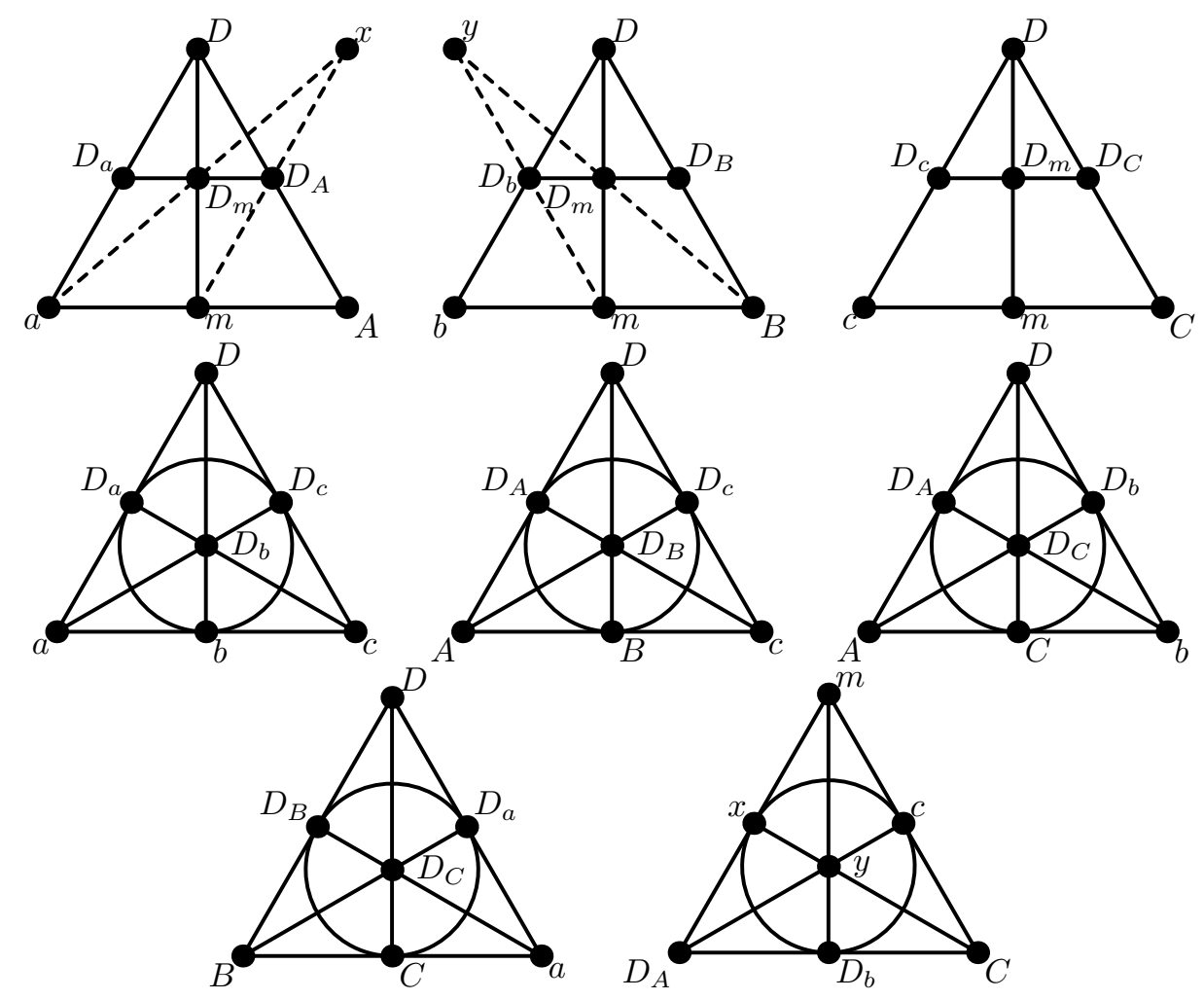

Figure 10: Configurations contained in $S$ in case where all the triples $\{A, a, D\},\{B, b, D\}$ and $\{C, c, D\}$ induce Steiner triple systems isomorphic to $S_{9}$, but $a, b$ and $D$ induce a Steiner triple system isomorphic to $S_{7}$. 
former holds if the induced Steiner triple system is isomorphic to $S_{7}$, the latter if it is isomorphic to $S_{9}$. Since the points $b, D_{m} \oplus b$ and $D \oplus b$ are distinct, the Steiner triple system induced by $D, A$ and $C$ must be isomorphic to $S_{7}$. By symmetry, the Steiner triple systems induced by $D, A$ and $B$, and $D, B$ and $C$ are also isomorphic to $S_{7}$.

Let $x=a \oplus D_{m}=m \oplus D_{A}$ and $y=B \oplus D_{m}=m \oplus D_{b}$. Consider now the Steiner triple system induced by the points $m, D_{A}$ and $D_{b}$. Since $D_{A} \oplus D_{b}=C$ (recall that the Steiner triple system induced by $A, C$ and $D$ is isomorphic to $S_{7}$ ), the point $x \oplus y$ is either $C$ (if the induced system is isomorphic to $S_{7}$ ) or $m \oplus C=c$ (if the induced system is isomorphic to $S_{9}$ ). Similarly, by considering the Steiner triple system induced by $D_{m}, a$ and $B$, we derive that $x \oplus y$ is either $C$ or $D_{m} \oplus C$. Since the points $C, m \oplus C$ and $D_{m} \oplus C$ are distinct, both the Steiner triple system induced by $m, D_{A}$ and $D_{b}$ and one induced by $D_{m}, a$ and $B$ are isomorphic to $S_{7}$ and $x \oplus y=C$. Hence, $D_{B}=D_{A} \oplus c=y$ but the points $y$ and $D_{B}$ must be distinct since they are contained in the copy of $S_{9}$ induced by the points $m, B$ and $D$.

We have shown that a non-projective Steiner triple system that is not a Hall triple system and such that every three independent points induce a Steiner triple system isomorphic to $S_{7}$ or $S_{9}$ cannot exist. The statement of the lemma now follows.

\section{Characterisations of affine Steiner triple sys- tems and Hall triple systems}

Before we can state our characterisation results, we have to show that every Steiner triple system containing $C_{A}$ also contains one of the configurations

$C_{S}^{1}$ and $C_{S}^{2}$. We prove instead that it homomorphically contains $C_{S}$ which is equivalent by Lemma 2 .

Lemma 6. Every Steiner triple system $S$ containing the anti-mitre configuration $C_{A}$ also homomorphically contains the squashed-square configuration $C_{S}$

Proof. Choose the points $\xi, \beta_{1}, \beta_{2}, \gamma_{1}$ and $\gamma_{2}$ to be the points of the antimitre configuration as depicted in Figure 11. Note that the five points are distinct and $\xi=\beta_{1} \oplus \beta_{2}=\gamma_{1} \oplus \gamma_{2}$. 


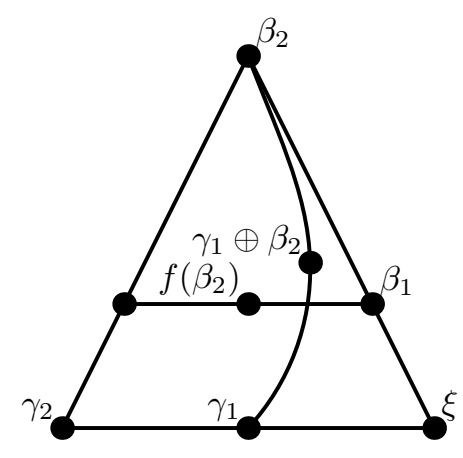

Figure 11: The notation used in the proof of Lemma 6.

For a point $z$ of a Steiner triple system $S$, let $f(z)=\left(z \oplus \gamma_{2}\right) \oplus \beta_{1}$ and $h(z)=\left(z \oplus \beta_{2}\right) \oplus \gamma_{1}$. Note that the functions $f$ and $h$ are not defined for all the points of $S$. More precisely, the function $f(z)$ is a bijection between its domain $D(f)=S \backslash\left\{\gamma_{2}, \gamma_{2} \oplus \beta_{1}\right\}$ and its image set $R(f)=S \backslash\left\{\beta_{1}, \gamma_{2} \oplus \beta_{1}\right\}$. Similarly, $h(z)$ is a bijection between $D(h)=S \backslash\left\{\beta_{2}, \beta_{2} \oplus \gamma_{1}\right\}$ and $R(h)=$ $S \backslash\left\{\gamma_{1}, \gamma_{1} \oplus \beta_{2}\right\}$.

Observe that if there exists a point $z$ of $S$ such that both $f(z)$ and $h(z)$ are defined and $f(z) \neq h(z)$, we are able to construct the squashed-square configuration in $S$. Indeed, we set $x_{0}=z, \alpha_{1}=z \oplus \gamma_{2}, \delta_{1}=z \oplus \beta_{2}$, $x_{\alpha \beta}=f(z)=\alpha_{1} \oplus \beta_{1}=\left(z \oplus \gamma_{2}\right) \oplus \beta_{1}$, and $x_{\gamma \delta}=h(z)=\delta_{1} \oplus \gamma_{1}=\left(z \oplus \beta_{2}\right) \oplus \gamma_{1}$; see Figure 4. Hence, in order to establish the statement of the lemma, it is enough to show that there exists a point $z$ of $S$ such that $f(z) \neq h(z)$.

Recall that $f$ and $h$ are defined on all the points of $S$ except $\beta_{2}, \gamma_{2}, \beta_{1} \oplus \gamma_{2}$ and $\beta_{2} \oplus \gamma_{1}$. The points $\beta_{2}$ and $\gamma_{2}$ are distinct from the remaining exceptional points, however, the points $\beta_{1} \oplus \gamma_{2}$ and $\beta_{2} \oplus \gamma_{1}$ might be the same. Analogously, the set of possible common values of $f$ and $h$ are the points of $S$ except for $\beta_{1}, \gamma_{1}, \beta_{1} \oplus \gamma_{2}$ and $\beta_{2} \oplus \gamma_{1}$. Again, these points are distinct with a possible exception of the pair of $\beta_{1} \oplus \gamma_{2}$ and $\beta_{2} \oplus \gamma_{1}$. Since the pairs of points that could be the same point are the same pairs both for the domains and image sets of $f$ and $h$, it holds that $|D(f) \cap D(h)|=|R(f) \cap R(h)|$.

Assume to the contrary that there is no point $z$ such that $z \in D(f) \cap D(h)$ and $f(z) \neq h(z)$. In particular, $f$ and $h$ restricted to $D(f) \cap D(h)$ are the same bijective mapping onto $R(f) \cap R(h)$. Therefore, the points of $D(f) \backslash D(h)$ are mapped by $f$ to $R(f) \backslash R(h)$. We conclude that $f\left(\beta_{2}\right)$ is one of the points $\gamma_{1}$ and $\gamma_{1} \oplus \beta_{2}$. However, $f\left(\beta_{2}\right)$ cannot be either of these two points; see 
Figure 11.

We can now state the theorem characterising affine and Hall Steiner triple systems.

Theorem 7. A Steiner triple system $S$ is affine if and only if it contains neither of the configurations $C_{16}, C_{S}^{1}$ or $C_{S}^{2}$. Moreover, if $S$ is known to be non-projective, then $S$ is affine if and only if it contains neither of the configurations $C_{S}^{1}$ and $C_{S}^{2}$.

Similarly, $S$ is a Hall triple system if and only if it contains neither of the configurations $C_{16}$ and $C_{A}$, and if $S$ is known to be non-projective, then $S$ is Hall if and only if it does not contain the configuration $C_{A}$.

Proof. It is easy to see that an affine Steiner triple system $S$ cannot contain either of the configurations $C_{16}, C_{S}^{1}$ and $C_{S}^{2}$. Suppose now that $S$ is not affine. If $S$ is projective, then it contains $C_{16}$. If $S$ is a Hall triple system, then it contains $C_{S}^{1}$ or $C_{S}^{2}$ by Lemmas 2 and 3. Finally, if $S$ is not projective and not a Hall triple system, then it contains $C_{A}$ by Lemma 5 , and hence it contains one of the configurations $C_{S}^{1}$ or $C_{S}^{2}$ by Lemmas 2 and 6 .

Similarly, a Hall triple system $S$ contains neither $C_{16}$ nor $C_{A}$ as neither of these two configurations is contained in $S_{9}$. Analogously to the above argument, a projective Steiner triple system $S$ contains the configuration $C_{16}$, and a non-projective Steiner triple system that is not a Hall triple system contains the configuration $C_{A}$ by Lemma 5 .

\section{Preliminary results on colourings with points of Steiner triple systems}

We now turn our attention to applying our characterisation results to edgecolourings of cubic graphs. We start with recalling several results on colourings of cubic graphs with Steiner triple systems. As the first, a theorem on edge-colourings of bridgeless cubic graphs with the points of Steiner triple systems from [8] is stated and proved. We provide its proof though the main idea follows that of [8] because of several reasons, the most important being that the proof is later altered to obtain edge-colourings in more special scenarios and we want to avoid extensive referring to a different paper. Another reason is that we want to give the proof using our notation. Given a subgraph $C$ of a graph $G$, let $G / C$ be the graph obtained by contracting the 


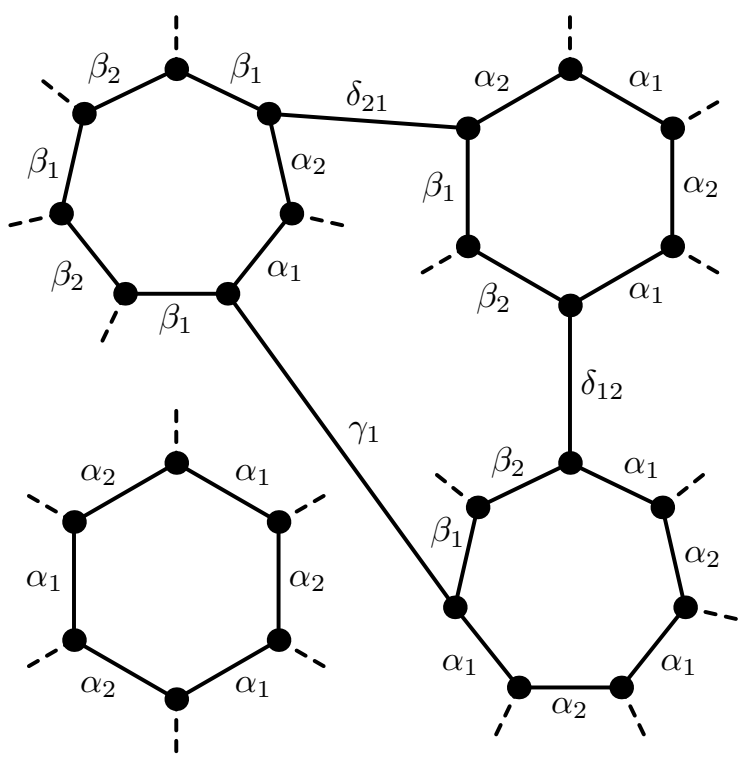

Figure 12: The colouring constructed in the proof of Theorem 8. The edges of $M \backslash\left(T_{1} \cup T_{2}\right)$ are drawn as dashed and coloured with the point $\xi$.

components of $C$ to single vertices, and removing the loops that may arise (but not the parallel edges).

Theorem 8 (Holroyd and Škoviera [8]). Let $S$ be a non-trivial Steiner triple system. Every bridgeless cubic graph is $S$-edge-colourable. Moreover, for every edge e of $G$ and every point $\xi$ of $S$, there exists an $S$-edge-colouring that assigns $\xi$ to $e$.

Proof. It can be shown using standard graph theory arguments that $G$ contains a perfect matching $M$ with the following properties (see $[9,10]$ for instance): $M$ contains the edge $e$ and the graph $H=G / C$, where $C$ is the complement of $M$ in $G$, contains two edge-disjoint spanning forests $T_{1}$ and $T_{2}$, e $\notin T_{1} \cup T_{2}$, such that the degree of a vertex $v$ is odd in $T_{i}, i=1,2$, if and only if the degree of $v$ in $H$ is odd. Such spanning forests are called parity forests.

Choose an arbitrary point $\alpha_{1}$ of $S$ distinct from $\xi$ and set $\alpha_{2}=\xi \oplus \alpha_{1}$. Let $\beta_{1}$ be a point of $S$ distinct from $\xi, \alpha_{1}$ and $\alpha_{2}$ and let $\beta_{2}=\xi \oplus \beta_{1}$. Further, let $\gamma_{i}=\alpha_{i} \oplus \beta_{i}$ for $i=1,2$. Finally, let $\delta_{12}=\alpha_{2} \oplus \beta_{1}$ and $\delta_{21}=\alpha_{1} \oplus \beta_{2}$; see Figure 13. 


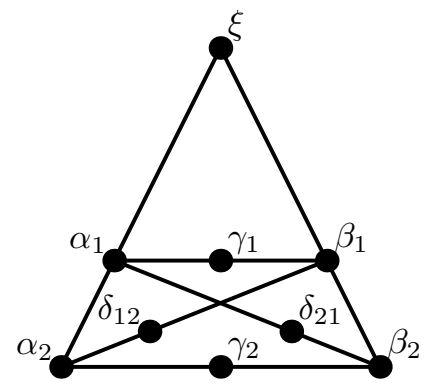

Figure 13: The notation used in the proof of Theorem 8.

If all the cycles of $C$ were even, we would colour the edges of $M$ with $\xi$ and the edges of the cycles with $\alpha_{1}$ and $\alpha_{2}$ alternately. This would give us the desired colouring of the edges of $G$. However, some cycles of $C$ could be odd. We cope with this in the rest of the proof.

The edges of cycles will be coloured with $\alpha$ 's and $\beta$ 's in such a way that the indices of the colours alternate precisely at vertices not incident with the edges of $T_{1}$. Once we choose which edges of a cycle $C_{1}$ of $C$ are coloured with $\alpha_{1}$ or $\beta_{1}$ and which with $\alpha_{2}$ or $\beta_{2}$, the points assigned to the edges of $T_{1}$ incident with $C_{1}$ are determined to be either $\gamma_{1}$ or $\gamma_{2}$ depending on the colours of the two edges incident with that edge of $T_{1}$. The choice of a colouring of a single cycle corresponding to a vertex of a component of $T_{1}$ determines the colourings of the edges of all cycles corresponding to the vertices of the same component of $T_{1}$. Since $T_{1}$ is acyclic and each cycle is incident with the number of edges of $T_{1}$ matching its parity, the edges of $T_{1}$ can be coloured with $\gamma_{1}$ and $\gamma_{2}$ in such a way that the colouring of $T_{1}$ can be extended to all cycles.

Similarly, we can partition the edges of $C$ into two classes such that the edges of one of the classes will be coloured with $\alpha_{1}$ or $\beta_{2}$, and the edges of the other class with $\alpha_{2}$ or $\beta_{1}$. The classes alternate at vertices not incident with an edge of $T_{2}$ and the edges of $T_{2}$ get the colours $\delta_{12}$ and $\delta_{21}$.

In the just described way, we have defined colourings of the edges of $T_{1}$ and $T_{2}$. The edges of $M$ not contained in $T_{1}$ or $T_{2}$ are coloured by $\xi$. As discussed above, the colouring of the edges of $M$ extends to the edges of $C$. Indeed, the colours of the edges of $T_{1}$ determine the indices and those of $T_{2}$ then completely fix the colours assigned to the edges of $C$. Since $e \in M \backslash\left(T_{1} \cup T_{2}\right)$, the colour of $e$ is $\xi$ as desired. The proof of the lemma is 
now completed.

Note that in addition to prescribing the colour of $e$ to be $\xi$, we can also assume in the proof of Theorem 8 that the colours of the edges of the even cycles corresponding to vertices that are isolated both in $T_{1}$ and $T_{2}$ are coloured with $\alpha_{1}$ and $\alpha_{2}$ alternately, and fix a colouring of one odd cycle.

We now sketch an alternative proof of the following result of Pál and Škoviera [11] — we focus on the aspects that are different and used in our further arguments. The proof was obtained during discussions of Zdeněk Dvořák and the first author.

Theorem 9 (Pál and Škoviera [11]). Every cubic graph with no bipartite end is $\mathrm{AG}(d, 3)$-edge-colourable for all $d \geq 3$.

The graph is first split into blocks with half-edges corresponding to bridges incident with the blocks. We next construct an edge-colouring of each block. It is required that the points assigned to each triple of edges sharing the same vertex form a triple in the system. In particular, the points assigned to a half-edge and the two edges incident with it form a triple. As stated in the next lemma, it is enough to be able to construct edge-colourings of nonbipartite ends and bridgeless cubic graphs since the half-edges of non-trivial blocks incident with more bridges can be merged to obtain graphs only of these two kinds.

Lemma 10. Let $H$ be a non-trivial block of a cubic graph incident with at least two half-edges. If $H$ is incident with an odd number of half-edges and $H$ is not a triangle with three half-edges, then the half-edges can be identified in such a way that the resulting graph is a non-bipartite end. On the other hand, if $H$ is incident with an even number of half-edges, then the half-edges of $H$ can be identified in such a way that the resulting graph (after a possible successive removal of pairs of parallel edges) is either a simple bridgeless cubic graph or a graph formed by a triple of parallel edges.

Proof. If $H$ is incident with four or more half-edges, than $H$ contains two halfedges $f_{1}$ and $f_{2}$ which are not incident with adjacent vertices. We identify the half-edges $f_{1}$ and $f_{2}$ and obtain a block with less half-edges and without parallel edges that also satisfies the assumption of the lemma. Hence, we assume in the rest that the block is incident with two or three half-edges.

If $H$ is incident with two half-edges, we identify the two half-edges and obtain a bridgeless cubic graph $H^{\prime}$ which need not be simple. We next 
remove pairs of parallel edges as we describe in Introduction: remove the pair of parallel edges and identify the two edges incident with the pair of parallel edges that remain in the graph. Note that the graph is kept being bridgeless and cubic in this way and thus we cannot obtain a loop. Hence, we either end with a simple bridgeless cubic graph or a triple of parallel edges as claimed in the statement of the lemma.

The case where $H$ is incident with three half-edges is more involved. We distinguish two cases based on whether or not the vertices of $H$ can be coloured with two colours. If the vertices can be coloured with two colours, then identify two half-edges incident with vertices of the same colour. This does not create a pair of parallel edges. In addition, the resulting graph is not a bipartite end since we have just created an odd cycle.

If the vertices of $H$ cannot be 2 -coloured, then identifying any pair of halfedges does not create a bipartite end. Hence, we have only to avoid creating a pair of parallel edges, i.e., we have to identify half-edges incident with nonadjacent vertices. This is possible unless $H$ is a triangle with incident three half-edges.

Every bridgeless cubic graph as well as a trivial block or a block formed by a triangle with three incident half-edges is $\mathrm{AG}(d, 3)$-edge-colourable [8]. Therefore, we only have to establish that each non-bipartite end is AG( $d, 3)$ edge-colourable for all $d \geq 3$ to finish the proof of Theorem 9. We next show that if the non-bipartite end is 3-edge-colourable, then it is indeed AG( $d, 3)$ edge-colourable for every $d \geq 2$.

Lemma 11. Let $S$ be a non-trivial affine Steiner triple system. Every 3edge-colourable non-bipartite end $G_{0}$ is $S$-edge-colourable.

Proof. Let $S$ be an affine Steiner triple system $\mathrm{AG}(d, 3), d \geq 2$. The points of $S$ are $d$-dimensional vectors $\left(x_{1}, \ldots, x_{d}\right)$ with $x_{i} \in\{0,1,2\}$. Let $G$ be the graph obtained from $G_{0}$ by suppressing the vertex incident with the halfedge and let $e$ be the new edge of $G$. For the rest of the proof, we fix a 3-edge-colouring of $G$.

We claim that $G$ contains an odd cycle $C_{0}$ passing through the edge $e$. Since $G$ is not bipartite, it contains an odd cycle $C$. If $e \in C_{0}$, the claim holds. Assume that $e \notin C_{0}$. Since $G$ is bridgeless, there exist two edgedisjoint paths in $G$ from the end-vertices of $e$ to $C$. The two paths combine with one of the two parts of $C$ delimited by the final vertices of the paths to an odd cycle $C_{0}$ containing the edge $e$. 
We are now ready to define the $S$-edge-colouring of $G_{0}$. The first coordinate $x_{1}$ is equal to 0,1 or 2 depending on the colour of the edge of $G$ in the fixed colouring. Note that the first coordinate of every two incident edges except for the two edges incident with the half-edge are distinct. The second coordinate $x_{2}$ is equal to 0 for all the edges not contained in $C_{0}$. For the other edges which form an even cycle in $G_{0}$, the second coordinate alternates between 1 and 2 . The remaining coordinates $x_{i}, i>2$, are equal to 0 . It is easy to verify that the defined colouring is an $S$-edge-colouring of $G_{0}$.

The proof that each non-3-edge-colourable (and thus non-bipartite) end is $\mathrm{AG}(d, 3)$-edge-colourable if $d \geq 3$, is obtained by combining arguments used in the proofs of Theorem 8 and Lemma 11. The first two coordinates are used to mimic the edge-colouring from the proof of Theorem 8 and the third coordinate to distinguish the colours assigned to the edges incident with the half-edge (in the same way as the second coordinate in the proof of Lemma 11). Since we do not use Theorem 9 in our further considerations and only use Lemmas 10 and 11, we leave the details missing to complete the proof of Theorem 9 to the reader.

\section{$7 \quad$ Non-3-edge-colourable ends}

By Lemma 10, the problem of an edge-colouring of a cubic graph can be decomposed into several problems dealing with edge-colourings of ends. In this section, we consider ends that are not 3-edge-colourable.

Lemma 12. Let $S$ be a Steiner triple system containing the configuration $C_{14}$. Every non-3-edge-colourable end $G_{0}$ is $S$-edge-colourable.

Proof. We alter the proof of Theorem 8. The points of $C_{14}$ are $\xi, \alpha_{i}, \beta_{i}$ and $\gamma_{i}$ as in Figure $1(a)$. Note that this is consistent with the notation used in the proof of Theorem 8 .

Let $e_{1}$ and $e_{2}$ be the two edges incident with the half-edge and $G$ be the bridgeless cubic graph obtained by suppressing the vertex incident with the half-edge. Let $e$ be the resulting edge of $G$. As in Theorem 8, we consider a perfect matching $M, e \in M$, and two disjoint parity forests $T_{1}$ and $T_{2}$ of $G / C$ where $C$ is the complement of $M$ and $e \notin T_{1} \cup T_{2}$. Let $v_{0}$ be the vertex of $G / C$ corresponding to a cycle incident with $e_{1}$.

Since the end is not 3-edge-colourable, $G / C$ contains a vertex of odd degree. Hence, both $T_{1}$ and $T_{2}$ contain some edges. Let $v_{0} \cdots v_{k}$ be a shortest 

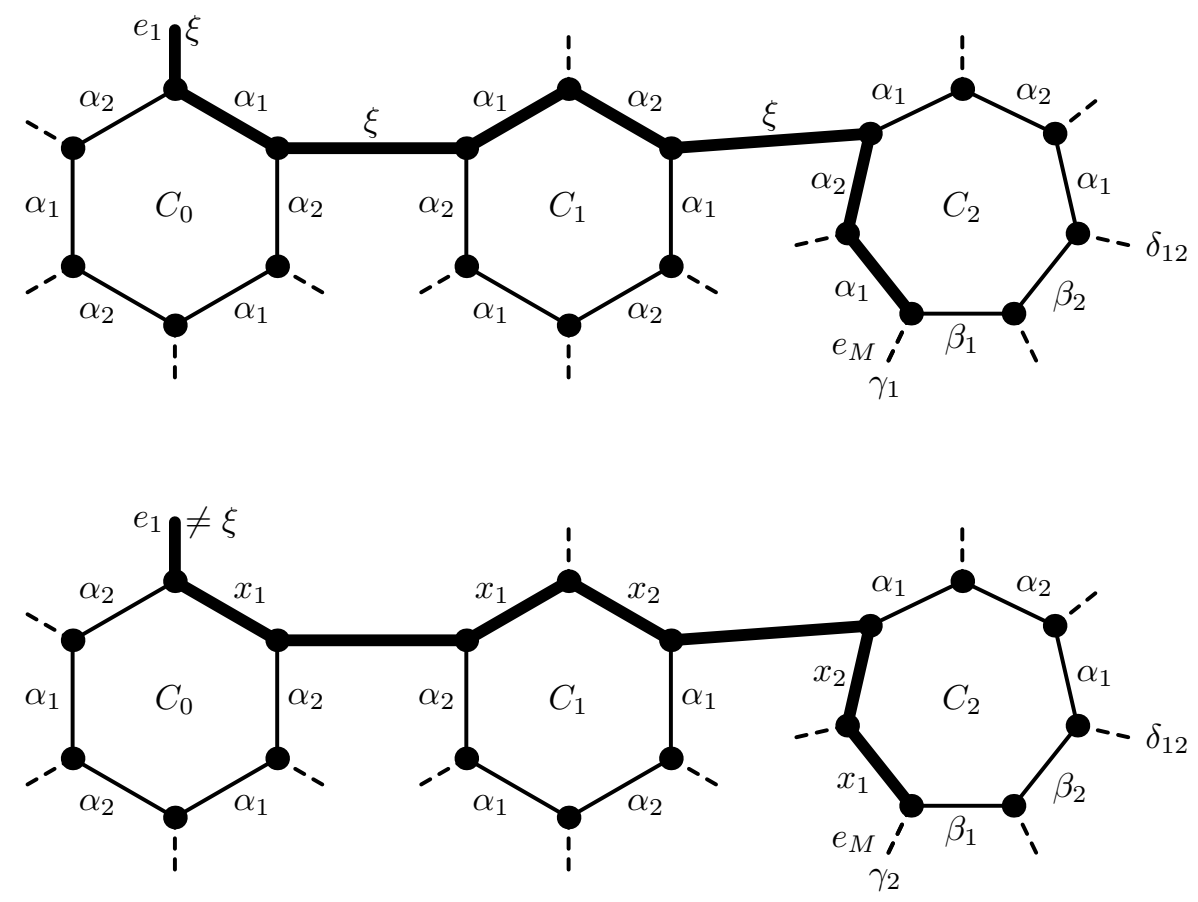

Figure 14: The modification of the colouring performed in the proof of Lemma 12-the original colouring is in the top and the modified in the bottom of the figure. The edges of the path $P$ are drawn solid bold.

path in $G / C$ from the vertex $v_{0}$ to a vertex $v_{k}$ that is not isolated in $T_{1}$ or $T_{2}$. Note that $k$ may be 0 . Further, let $C_{i}$ be the cycle of $C$ corresponding to the vertex $v_{i}, i=0, \ldots, k$, and let $P$ be the path in $G_{0}$ comprised of the edge $e_{1}$, the edges corresponding to the path $v_{0} \cdots v_{k}$ and parts of the cycles $C_{0}, \ldots, C_{k}$. By symmetry, we can assume that $e_{2}$ does not belong to $P$, see Figure 14. The last vertex of $P$ is the only vertex of $P$ incident with an edge contained in $T_{1} \cup T_{2}$.

Next, we modify the colouring obtained in the proof of Theorem 8. As mentioned after its proof, we can assume that the edges of $P \cap C$ are coloured with $\alpha_{1}$ and $\alpha_{2}$ only. In addition, we can assume that the colours of edges of $P \cap C$ incident with the same edge of $P \cap M$ are the same, i.e., they both are either $\alpha_{1}$ or $\alpha_{2}$, the last edge of $P$ is coloured with $\alpha_{1}$ and the edge $e_{M}$ of $M$ incident with the last vertex of $P$ is contained in $T_{1}$ (see the figure). In particular, the colour of $e_{M}$ is $\gamma_{1}$. Note that all the edges of $P \cap M$ are 
coloured with $\xi$.

Let $x_{1}$ be the point $\beta_{1} \oplus \gamma_{2}$. Note that $x_{1}$ is distinct from the points $\xi$, $\alpha_{1}$ and $\alpha_{2}$. Further, let $x_{2}=\xi \oplus x_{1}$. Analogously, $x_{2}$ is different from $\alpha_{1}$ and $\alpha_{2}$. Note that $\alpha_{2}+x_{i} \neq \xi$ for each $i \in\{1,2\}$.

We now alter the constructed colouring. Recolour the edge $e_{M}$ to $\gamma_{2}$ and swap the indices of the colours of the edges of the subtree of $T_{1}$ separated by $e_{M}$ from $v_{k}$, i.e., the edges of the subtree coloured with $\gamma_{1}$ are now coloured with $\gamma_{2}$ and vice versa. This results in a change of the colouring of the edges of $C$ contained in the cycles incident with edges of swapped colours. By the choice of the path $v_{0} \cdots v_{k}$, the colours of all the edges of $P$ as well as edges incident with them except for the edge $e_{M}$ have been preserved.

We now modify the colouring of the edges of $P$ in such a way that the obtained colouring is an $S$-edge-colouring of $G_{0}$. The colours of the edges of $P \cap C$ that are originally coloured with $\alpha_{i}$ are changed to $x_{i}$ both for $i=1$ and $i=2$. Consider now an edge $f \in P \cap M$. Note that both the ends of $f$ are either incident with edges coloured $\alpha_{1}$ and $x_{2}$, or with edges coloured $\alpha_{2}$ and $x_{1}$. Hence, we can extend the colouring to the edges of $P \cap M$. Since the colour of $e_{1}$ is not equal to $\xi$, and the colour of $e_{2}$ is $\xi$, the colouring can also be extended to the half-edge of $G_{0}$ which is incident with $e_{1}$ and $e_{2}$. This finishes the proof of the lemma.

\section{3-edge-colourable ends}

In Section 7, we constructed colourings of non-3-edge-colourable ends. It remains to consider 3-edge-colourable ends. It seems that the core of the problem lies in hamiltonian ends. More precisely, we first construct the desired colourings for hamiltonian ends and we later show how to reduce the problem of colouring general 3-edge-colourable ends to hamiltonian ones. The first case we consider are hamiltonian non-bipartite ends.

Lemma 13. Let $S$ be a Steiner triple system containing the configuration $C_{14}$. Every hamiltonian non-bipartite end $G_{0}$ is $S$-edge-colourable. Moreover, there exists an $S$-edge-colouring such that the edges not contained in the Hamilton cycle receive at most three distinct colours.

Proof. Let $G$ be the graph obtained from $G_{0}$ by suppressing the vertex incident with the half-edge, $e$ the resulting edge of $G$, and $e_{1}$ and $e_{2}$ the two edges of $G_{0}$ incident with the half-edge. Further, let $C_{H}$ be the Hamilton 


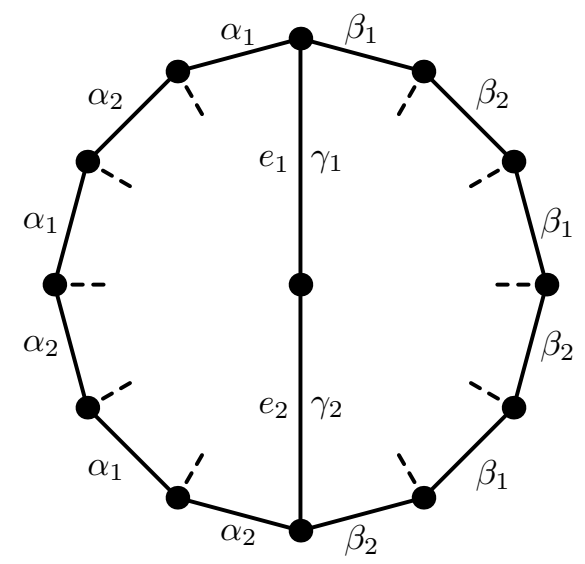

Figure 15: The colouring of $G_{0}$ constructed in the proof of Lemma 13 in the case where the cycle $C_{e}$ is odd in $G$. The dashed chords are labelled with $\xi$.

cycle with $e \notin C_{H}$ and for every chord $f$ of $C_{H}$, let $C_{f}$ be one of the cycles formed by $f$ and a part of the cycle $C_{H}$. Since the cycles $C_{H}$ and $C_{f}$ form a base of the cycle space of $G$ and $G$ is not bipartite, one of these cycles is odd. Since $G$ is cubic, the length of $C_{H}$ is even and thus one of the cycles $C_{f}$ is odd.

We first consider the case that the cycle $C_{e}$ is odd (in the graph $G$ ). The edges $e_{1}$ and $e_{2}$ are coloured with $\gamma_{1}$ and $\gamma_{2}$, the other chords of $C_{H}$ are coloured with $\xi$. The edges of one of the parts of $C_{H}$ delimited by $e$ are coloured with $\alpha_{1}$ and $\alpha_{2}$ and the edges of the other part with $\beta_{1}$ and $\beta_{2}$ (see Figure 15). Let us remind that the points of $C_{14}$ are $\xi, \alpha_{i}, \beta_{i}$ and $\gamma_{i}$ as in Figure 1 $(a)$.

In the rest of the proof, we assume that the cycle $C_{e}$ is even and consider a cycle $C_{f}, f \neq e$, that is odd. Before we proceed further, let us introduce some additional notation: $\alpha_{1}^{\prime}$ is the point $\alpha_{2} \oplus \gamma_{1}, \alpha_{2}^{\prime}$ is $\xi \oplus \alpha_{1}^{\prime}$, and $\xi^{\prime}$ is $\alpha_{2}^{\prime} \oplus \beta_{2}$. Since $\alpha_{1}^{\prime} \neq \xi$, the point $\alpha_{2}^{\prime}$ is well-defined. Moreover $\alpha_{2}^{\prime} \neq \beta_{2}$, i.e., the point $\xi^{\prime}$ is also well-defined.

We now distinguish two cases based on whether the chords $e$ and $f$ cross. If the chords $e$ and $f$ cross (see Figure 16(a)), colour $f$ with $\gamma_{1}$ and one of the parts of $C_{H}$ delimited by $f$, say one incident with $e_{1}$, with $\alpha_{1}$ and $\alpha_{2}$ alternately. The other part of $C_{H}$ is split by $e_{2}$ into two parts: the edges of one of the parts are coloured with $\beta_{1}$ and $\beta_{2}$ alternately and the edges of the other with $\alpha_{1}^{\prime}$ and $\alpha_{2}^{\prime}$ alternately. Finally, the edges $e_{1}$ and $e_{2}$ are coloured 


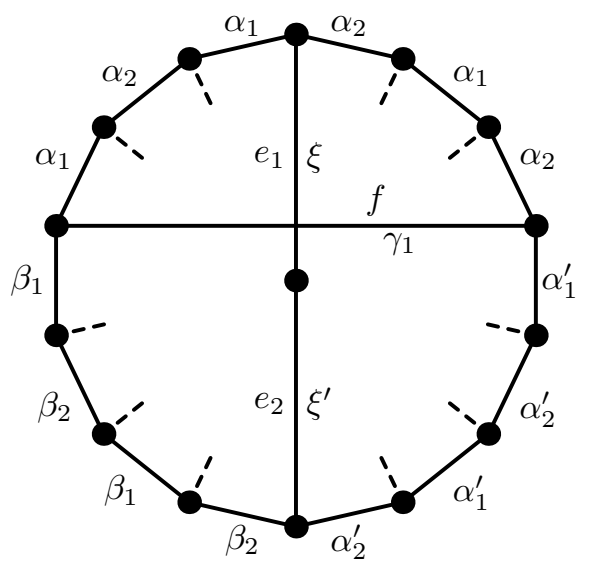

(a)

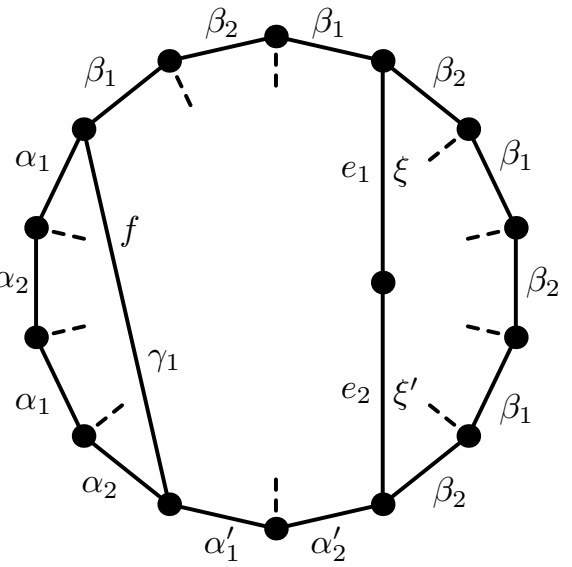

(b)

Figure 16: The colourings of $G_{0}$ constructed in the proof of Lemma 13 in the case where the cycle $C_{e}$ is even and $(a)$ the chords $e$ and $f$ cross, $(b)$ the chords $e$ and $f$ do not cross. The dashed chords are labelled with $\xi$.

with $\xi$ and $\xi^{\prime}$, and the remaining chords of $C_{H}$ are coloured with $\xi$. It is straightforward to verify that the colouring is an $S$-edge-colouring. Since $\xi \neq \xi^{\prime}$, the colouring can be extended to the half-edge.

The final case to consider is that the chords $e$ and $f$ do not cross. By the symmetry, we can assume that the part of $C_{H}$ delimited by $e_{1}$ and $f$ is odd and the part delimited by $e_{2}$ and $f$ is even; see Figure 16(b). The edges of the part of $C_{H}$ between $f$ and $e_{1}$, and $e_{1}$ and $e_{2}$, are coloured with $\beta_{1}$ and $\beta_{2}$ alternately. The edge $f$ is assigned $\gamma_{1}$ and the edges of the part of $C_{H}$ delimited by $f$ are assigned $\alpha_{1}$ and $\alpha_{2}$ alternately. The remaining edges of $C_{H}$ are then coloured with $\alpha_{1}^{\prime}$ and $\alpha_{2}^{\prime}$. Finally, the edge $e_{2}$ is coloured with $\xi^{\prime}$ and the remaining chords of $C_{H}$ with $\xi$. Since $\xi \neq \xi^{\prime}$, this colouring can also be extended to the half-edge.

Note that, regardless whether the cycle $C_{e}$ is odd or even, there are three distinct points assigned to the edges not contained in the Hamilton cycle, namely, the points $\gamma_{1}, \xi$ and $\xi^{\prime}$.

It remains to consider hamiltonian bipartite ends. This is the point where we will utilise our characterisation results since such ends cannot be edgecoloured by affine Steiner triple systems. 


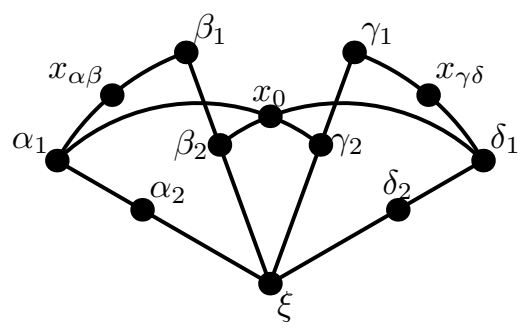

Figure 17: The squashed-square configuration $C_{S}$ enhanced by two new points $\alpha_{2}=\xi \oplus \alpha_{1}$ and $\delta_{2}=\xi \oplus \delta_{1}$. Note that it is required that $x_{\alpha \beta} \neq x_{\gamma \delta}$ but the other pairs of points can coincide.

Lemma 14. Let $S$ be a Steiner triple system homomorphically containing the squashed-square configuration $C_{S}$. Every hamiltonian bipartite end $G_{0}$ is $S$-edge-colourable. Moreover, there exists an $S$-edge-colouring such that the edges not contained in the Hamilton cycle receive at most five distinct colours.

Proof. Let us enhance the squashed-square configuration $C_{S}$ by introducing two new points $\alpha_{2}=\xi \oplus \alpha_{1}$ and $\delta_{2}=\xi \oplus \delta_{1}$; see Figure 17. Let $G$ be the graph obtained from $G_{0}$ by suppressing the vertex incident with the half-edge, $e$ the resulting edge of $G$, and $e_{1}$ and $e_{2}$ the two edges of $G_{0}$ incident with the half-edge. Further, let $C_{H}$ be the Hamilton cycle of $G$ with $e \notin C_{H}$.

Assume first that $G$ contains a chord $f$ that crosses $e$ as depicted in Figure 18. The edge $e_{1}$ is coloured with $x_{\alpha \beta}, e_{2}$ with $x_{\gamma \delta}$, and $f$ with $x_{0}$. The other chords of $C_{H}$ are coloured with $\xi$. The edges of the parts of $C_{H}$ delimited by $e$ and $f$ are coloured with $\alpha_{1}$ and $\alpha_{2}, \beta_{1}$ and $\beta_{2}, \gamma_{1}$ and $\gamma_{2}$, and $\delta_{1}$ and $\delta_{2}$, each part with one of the pairs of the colours alternately. Since $x_{\alpha \beta} \neq x_{\gamma \delta}$, the colouring can be extended to the half-edge. Note that only the points $\xi, x_{0}, x_{\alpha \beta}$ and $x_{\gamma \delta}$ are assigned to the edges not contained in the Hamilton cycle.

A more involved case is when no chord of $C_{H}$ crosses $e$. Since $G$ has no parallel edges (with a possible exception of $e$ ), it contains a pair of crossing chords. Each pair of crossing chords split $C_{H}$ into four parts, out of which one is incident with both the end-vertices of $e$. Choose among all pairs of chords $f_{1}$ and $f_{2}$ the one with distance between the end-vertices of $f_{1}$ and $f_{2}$ delimiting the part opposite to the part incident with $e$ the smallest possible. 


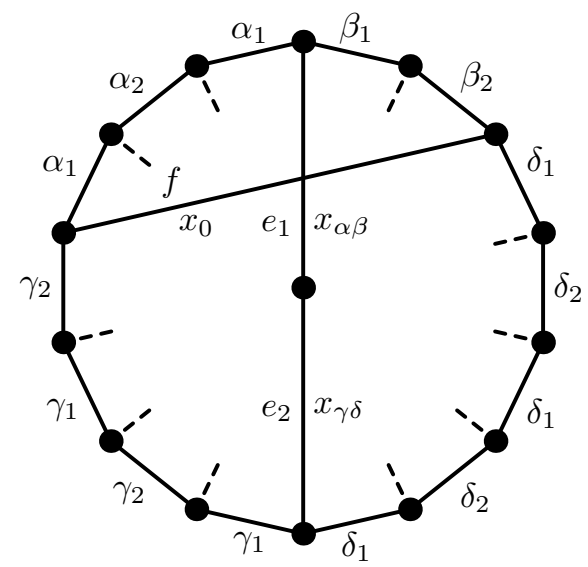

Figure 18: The colouring of $G_{0}$ constructed in the proof of Lemma 14 in the case where there is a chord crossing $e$. The dashed chords are labelled with $\xi$.
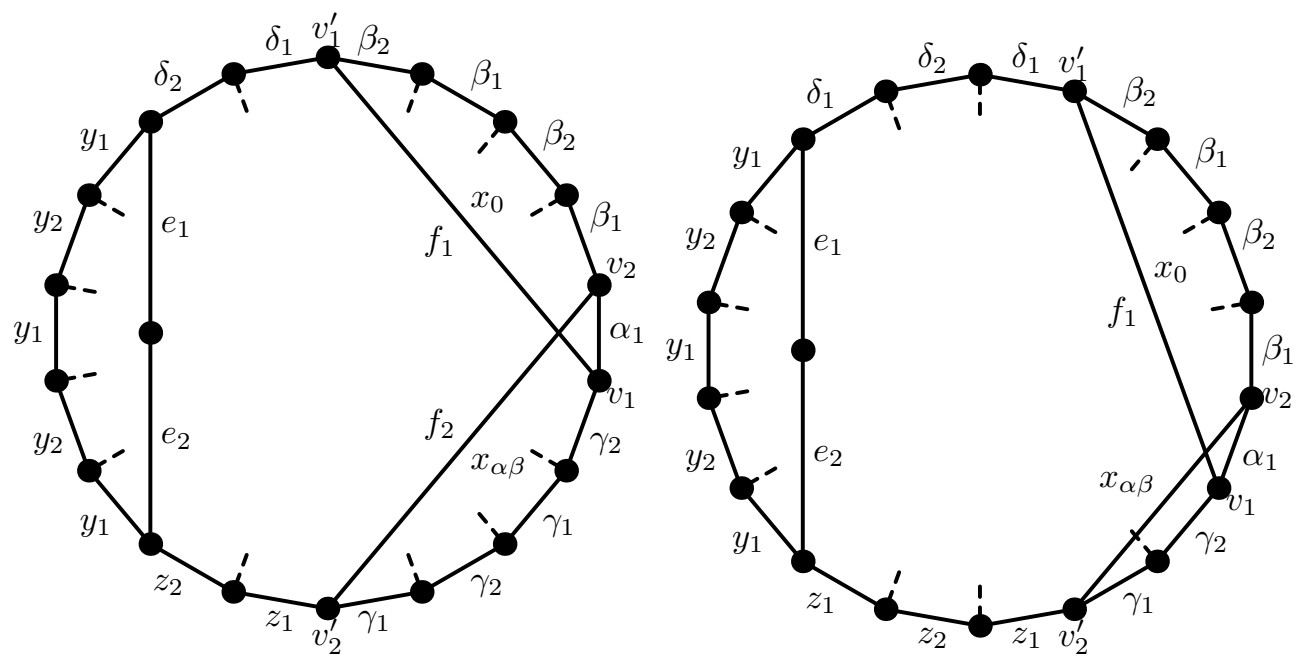

Figure 19: The colourings of $G_{0}$ constructed in the proof of Lemma 14 in the case where there is no chord crossing $e$. The dashed chords are labelled with $\xi$. 
It is not hard to show that this distance must be equal to one, otherwise, there would exist a pair of crossing chords with closer end-vertices. Let $v_{1}$ and $v_{2}$ be the end-vertices of $f_{1}$ and $f_{2}$ that are adjacent and $v_{1}^{\prime}$ and $v_{2}^{\prime}$ the other end-vertices of $f_{1}$ and $f_{2}$.

Before we proceed further, we show that it can be assumed that $x_{\alpha \beta} \neq \gamma_{1}$ or $x_{\gamma \delta} \neq \beta_{1}$ (note that the two inequalities are symmetric). If $x_{\alpha \beta}=\gamma_{1}$ and $x_{\gamma \delta}=\beta_{1}$, then $\alpha_{1}=\delta_{1}$; see Figure 17. Consequently, $\beta_{2}=\gamma_{2}$ and $\beta_{1}=\gamma_{1}$. However, this implies $x_{\alpha \beta}=x_{\gamma \delta}$ which is inconsistent with the definition of the configuration $C_{S}$. Hence, we can assume that $x_{\alpha \beta} \neq \gamma_{1}$ in the rest of the proof. Since $x_{\alpha \beta} \neq \gamma_{1}$, there exists a point $z_{1}$ that forms a triple with $x_{\alpha \beta}$ and $\gamma_{1}$. Observe that $z_{1} \neq \xi$ (otherwise, $x_{\alpha \beta}$ would be equal to $\gamma_{2}, x_{0}$ would be $\beta_{1}$ and $\xi$ would be $\delta_{1}$ which is impossible). Consequently, there exists a point $z_{2}=\xi \oplus z_{1}$.

We are now ready to colour the edges of $G$. The edge $v_{1} v_{2}$ is coloured with $\alpha_{1}$, the edge $f_{1}$ with $x_{0}$ and the edge $f_{2}$ with $x_{\alpha \beta}$. The chords of $C_{H}$ distinct from $e_{1}, e_{2}, f_{1}$ and $f_{2}$ are coloured with $\xi$. The edges of the part of $C_{H}$ delimited by $v_{1}$ and $v_{2}^{\prime}$ are coloured with $\gamma_{1}$ and $\gamma_{2}$ alternately and the edges of the part delimited by $v_{2}$ and $v_{1}^{\prime}$ are coloured with $\beta_{1}$ and $\beta_{2}$ as in Figure 19. This colouring switches at the vertex incident with $f_{1}$ to an alternating colouring with $\delta_{1}$ and $\delta_{2}$ until it hits the end-vertex of the edge $e_{1}$. The edges of $C_{H}$ between the end-vertices of $e_{2}$ and $f_{2}$ are coloured with $z_{1}$ and $z_{2}$ alternately; see Figure 19.

It remains to colour the edges of the part of $C_{H}$ delimited by $e$ and the edges $e_{1}$ and $e_{2}$ themselves. The coloured edge incident with $e_{1}$ is coloured with $\delta_{k}$ and the coloured edge incident with $e_{2}$ is coloured with $z_{k}$ (note that the indices of the two colours are the same by the parity constraints). We assert that $\delta_{k} \neq z_{k}$ : if $z_{1}=\delta_{1}$, then $x_{\alpha \beta}=x_{\gamma \delta}$ which is impossible. If $z_{2}=\delta_{2}$, then $z_{1}=\delta_{1}$ which we have just excluded.

Choose arbitrary a point $y_{1}$ of $S$ that is distinct from $\xi, \delta_{k}$ and $z_{k}$, and let $y_{2}=\xi \oplus y_{1}$. The remaining edges of $H_{C}$ are coloured with $y_{1}$ and $y_{2}$ alternately in such a way that the edges $e_{1}$ and $e_{2}$ are incident with edges coloured with $y_{1}$. Since $y_{1} \notin\left\{z_{k}, \delta_{k}\right\}$, the colouring can be extended to $e_{1}$ and $e_{2}$. Moreover, since $z_{k} \neq \delta_{k}$, the colours of $e_{1}$ and $e_{2}$ are distinct. Hence, the colouring can also be extended to the half-edge.

It remains to verify that there are at most five points used to colour the edges not contained in the Hamilton cycle: indeed, only the points $\xi, x_{0}, x_{\alpha \beta}$ and the points assigned to $e_{1}$ and $e_{2}$ are such points. The proof of the lemma is now finished. 
We have constructed colourings of hamiltonian ends. A 3-edge-colourable end does not need to be hamiltonian in general but we can reduce the problem to hamiltonian ends as described in the next lemma.

Lemma 15. Let $S$ be a Steiner triple system containing the configuration $C_{14}$ and homomorphically containing the squashed-square configuration $C_{S}$. Every 3-edge-colourable end $G_{0}$ is $S$-edge-colourable.

Proof. Let $G$ be the graph obtained by suppressing the vertex $u$ incident with the half-edge and $e=v_{0} v_{1}$ the resulting edge. Fix a colouring of the edges of $G$ with three colours, say red, green and blue, such that the edge $e$ is red and the cycle formed by green and blue edges that contains $v_{0}$ is the longest possible.

Let $w_{1}, \ldots, w_{k}$ be the vertices of the cycle formed by green and blue edges that contains the vertex $v_{0}$ and assume that $w_{1}=v_{0}$. We construct an auxiliary end $H_{0}$ with the vertices $w_{0}, \ldots, w_{k}$ where $w_{0}$ is a new vertex which will be incident with the half-edge of $H_{0}$. The cycle $w_{1} \cdots w_{k}$ is contained in $H_{0}$. In addition, the vertices $w_{i}$ and $w_{j}$ are joined in $H_{0}$ by an edge $e_{i j}$ if $G$ contains a path $P_{i j}$ formed by red and blue edges such that $w_{i}$ and $w_{j}$ are the only vertices of $P_{i j}$ contained in the cycle $w_{1} \cdots w_{k}$. Note that for each vertex $w_{i}$, there exists a unique vertex $w_{j}$ with this property. The chord incident with $w_{1}$ is replaced by a path containing the vertex $w_{0}$. Observe that the resulting graph $H_{0}$ is a hamiltonian end. In addition, $H_{0}$ has no parallel edges: if two vertices $w_{i}$ and $w_{j}$ adjacent in the cycle were joined by a chord, then we could assume by the symmetry that the edge $w_{i} w_{j}$ of the cycle is blue and switch the red and blue colours on the cycle $P_{i j} \cup\left\{w_{i} w_{j}\right\}$ thereby obtaining a longer green-blue cycle with $v_{0}$. This would contradict our choice of the 3-edge-colouring.

By Lemma 13 or 14 (depending on whether $H_{0}$ is bipartite), $H_{0}$ has an $S$ edge-colouring such that at most five distinct points of $S$ are used to colour the chords of the Hamilton cycle of $H_{0}$. Choose a point $\xi_{0}$ of $S$ distinct from the five points used on edges not contained in the Hamilton cycle. In addition, choose another point $\alpha_{0}$ distinct from $\xi_{0}$. We now construct an $S$-edge-colouring of the edges of $G_{0}$. The edges of the cycle $w_{1} \cdots w_{k}$ keep their colours. The red edges of the path $P_{i j}$ get the colour of the edge $e_{i j}$; the edge $w_{1} u$ gets the colour of $w_{0} w_{1}$, and the red edges on the path from $w_{1}$ as well as the edge $u v_{1}$ get the colour of the other edge incident with $w_{0}$. The remaining red edges of $G_{0}$ are coloured with $\alpha_{0}$. The green edges of $G_{0}$ not contained in the cycle $w_{1} \cdots w_{k}$ are coloured with $\xi_{0}$. 
The only edges that are not yet coloured are blue edges not contained in the cycle $w_{1} \cdots w_{k}$. Now observe that each end-vertex of every uncoloured blue edge is incident with two edges coloured with the same pair of points of $S$ (one of these two colours being $\xi_{0}$ ). Hence, the colouring can be extended to all the edges of $G_{0}$. This finishes the proof of the lemma.

\section{Characterisation of edge-colourability of cu- bic graphs}

In Sections 7 and 8, we constructed $S$-edge-colourings of ends of cubic graphs. It remains to combine these colourings to a colouring of the whole cubic graph.

Theorem 16. Let $G$ be a cubic graph and $S$ a non-trivial point-transitive Steiner triple system.

- If $S$ is projective, then $G$ is $S$-edge-colourable if and only if $G$ is bridgeless.

- If $S$ is affine, then $G$ is $S$-edge-colourable if and only if $G$ has no bipartite end.

- If $S$ is neither projective nor affine, then $G$ is always $S$-edge-colourable.

Proof. Fix a cubic graph $G$ and a non-trivial point-transitive Steiner triple system $S$. If $S$ is projective, then $G$ is $S$-edge-colourable if and only if $G$ is bridgeless [8].

Next, we assume $S$ is affine. If $G$ has a bipartite end, then $G$ is not $S$-edgecolourable [8]. Hence, assume $G$ has no bipartite ends. If $G$ is bridgeless, $G$ is $S$-edge-colourable since all bridgeless cubic graphs are edge-colourable by all non-trivial Steiner triple systems; see Theorem 8. Hence, we also assume $G$ has one or more bridges.

Split now the graph $G$ along its bridges into several blocks. We now colour each block with its incident half-edges. Since $S$ is affine, it contains the configuration $C_{14}$. Hence, all the ends can be $S$-edge-coloured either by Lemma 11 or by Lemma 12. Let us now consider a block incident with two or more half-edges. The trivial blocks and the blocks formed by a triangle with three half-edges are clearly $S$-edge-colourable. By Lemma 10, the half-edges 
of the remaining blocks of $G$ can be identified in such a way that the resulting graph (after a possible removal of parallel edges) is a bridgeless cubic graph, a triple edge or a non-bipartite end. All such graphs are $S$-edge-colourable as argued before. Since the obtained $S$-edge-colouring can be extended to the removed parallel edges, the original block of $G$ is also $S$-edge-colourable.

It remains to combine the colourings of the blocks. After contracting the blocks of $G$ into single vertices, the graph $G$ becomes a tree. We further refer to the vertices as nodes in order to distinguish them from the vertices of $G$. Root this tree at any of its nodes. We fix the $S$-edge-colourings of the blocks from the root of the tree to its leaves. The block corresponding to the root keeps its original colouring. Since $S$ is point-transitive, we can permute the colourings of the blocks corresponding to the nodes adjacent to the root (its children) in such a way that they match on the half-edges. Next, we permute the edge-colourings of the blocks corresponding to the grand-children in such a way that they match the colours of edges leading from the blocks corresponding to the children. We proceed in this way until we obtain an $S$-edge-colouring of the entire graph $G$.

It remains to consider the case where $S$ is neither projective nor affine. By Theorems 1 and 7, and Lemma 2, we deduce that $S$ contains the configuration $C_{14}$ as well as it homomorphically contains the squashed-square configuration $C_{S}$. If $G$ is bridgeless, $G$ is $S$-edge-colourable by Theorem 8. Otherwise, we also split $G$ along its bridges to blocks. The ends can be $S$-edge-coloured by Lemmas 12 and 15. The trivial blocks and the blocks formed by triangles with three half-edges are $S$-edge-colourable from trivial reasons. The half-edges of the remaining blocks can be identified to get bridgeless graphs and (nonbipartite) ends. Hence, such blocks are also $S$-edge-colourable. The obtained colourings of the blocks of $G$ can be combined to an $S$-edge-colouring of the whole graph $G$ in the same way as in the case of affine Steiner triple systems.

As a corollary of Theorem 16, we derive the conjecture of Holroyd and Škoviera (Conjecture 1) for point-transitive Steiner triple systems.

Corollary 17. Let $G$ be a cubic graph and $S$ a non-projective (and thus non-trivial) point-transitive Steiner triple system. If $G$ has no bipartite end or $S$ is not affine, then $G$ is $S$-edge-colourable. 


\section{Concluding remarks}

In the first part of the paper, we have characterised several important classes of Steiner triple systems in terms of forbidden subconfigurations: we have shown that a Steiner triple system is affine if and only if it contains neither of the configurations $C_{16}, C_{S}^{1}$ or $C_{S}^{2}$, and $S$ is a Hall triple system if and only if it contains neither the configuration $C_{16}$ nor $C_{A}$. It seems to be interesting to provide such characterisations of other important classes of Steiner triple systems.

Let us now turn our attention to edge-colourings of cubic graphs with elements of Steiner triple systems. Conjecture 1 is now proven for affine Steiner triple systems since all affine Steiner triple systems are point-transitive. Hence, it is only open whether every non-projective non-affine Steiner triple system that is not point-transitive is universal. The smallest such Steiner triple system has 13-vertices; let $S_{13}^{\prime}$ be this Steiner triple system. Note that there are only two Steiner triple systems with 13 vertices, namely the point-transitive system $S_{13}$ mentioned in Section 1, and the system $S_{13}^{\prime}$.

Along the lines of the proof of Theorem 16, we tried to establish that $S_{13}^{\prime}$ is also universal. First we identified several configurations such that if all of them are present in $S_{13}^{\prime}$, all the ends can be coloured. As in the proof of the lemmas in Sections 7 and 8, several cases based on whether the end is 3-colourable, bipartite, etc., need to be considered. Next, we prepared a computer program to check the presence of these configurations in $S_{13}^{\prime}$ with the additional requirement that one of their points, the one to be assigned to the half-edge, is a prescribed point of $S_{13}^{\prime}$. In this way, the presence of all the needed configurations in $S_{13}^{\prime}$ with the special point being any of the points of $S_{13}^{\prime}$ was established.

Based on this, we concluded that each block of every cubic graph can be $S_{13}^{\prime}$-edge-coloured if one of the half-edges incident with it is precoloured with a point of $S_{13}^{\prime}$. Hence, the system $S_{13}^{\prime}$ is universal. We believe that an analogous argument can be used to establish that other small non-pointtransitive Steiner triple systems are universal but we did not yet manage to develop any theoretical background that would give us any hope of extending our results to Steiner triple systems that need not be point-transitive. 


\section{Acknowledgement}

The second author thanks the Department of Applied Mathematics of Charles University for hospitality during her stay in Prague when this research was conducted. All the authors are indebted to Zdeněk Dvořák for valuable discussions on edge-colourings of cubic graphs with affine Steiner triple systems as well as to Alexander Rosa for his insights on the structure of Steiner triple systems, in particular, for pointing out the counting argument that $C_{14}$ is a forbidden configuration for projective Steiner triple systems.

\section{References}

[1] D. Archdeacon: Generalizations of Tait coloring cubic graphs, in: Problems in topological graph theory, http: //www. emba.uvm.edu/〜archdeac/problems/problems.html.

[2] C. J. Colbourn, A. Rosa: Triple systems, Oxford Univ. Press, Oxford, 1999.

[3] H.-L. Fu: A generalization of Tait coloring cubic graphs, Bull. Inst. Combin. Appl. 31 (2001), 45-49.

[4] M. J. Grannell, T. S. Griggs, M. Knor, M. Škoviera: A Steiner triple system which colours all cubic graphs, J. Graph Theory 46 (2004), 1524 .

[5] M. J. Grannell, T. S. Griggs, E. Mendelsohn: A small basis for fourline configurations in Steiner triple systems, J. Combin. Des. 3 (1995), $51-59$.

[6] T. S. Griggs, E. Máčajová: personnal communication.

[7] M. Hall, Jr.: Automorphisms of Steiner triple systems, IBM J. Res. Develop. 4(5) (1960), 460-472.

[8] F. C. Holroyd, M. Škoviera: Colouring of cubic graphs by Steiner triple systems, J. Combin. Theory Ser. B. 91 (2004), 57-66.

[9] D. Král', E. Máčajová, O. Pangrác, A. Raspaud, J.-S. Sereni, M. Škoviera: Projective, affine and abelian colorings of cubic graphs, to appear in European Journal of Combinatorics. 
[10] T. Kaiser, D. Král', S. Norine: Unions of perfect matchings in cubic graphs, in M. Klazar, J. Kratochvíl, J. Matoušek, R. Thomas, P. Valtr (eds.): Topics in Discrete Mathematics, Springer, 2006, 225-230.

[11] D. Pál, M. Škoviera: Colouring cubic graphs by small Steiner triple systems, to appear in Graphs Combin.

[12] D. R. Stinson, Y. J. Wei: Some results on quadrilaterals in Steiner triple systems, Discrete Math. 105 (1992), 207-219.

[13] L. Teirlinck: On linear spaces in which every plane is either projective or affine, Geom. Dedicata 4 (1975), 39-44.

[14] V. G. Vizing: Colouring the vertices of a graph with prescribed colours (in Russian), Metody Diskretnogo Analiza Teorii Kodov i Skhem 29 (1976), 3-10. 